\title{
Global characteristics of GRBs observed with INTEGRAL and the inferred large population of low-luminosity GRBs ${ }^{\star, \star \star}$
}

\author{
S. Foley ${ }^{1}$, S. McGlynn ${ }^{1}$, L. Hanlon ${ }^{1}$, S. McBreen ${ }^{2}$, and B. McBreen ${ }^{1}$ \\ 1 UCD School of Physics, University College Dublin, Dublin 4, Ireland \\ e-mail: sfoley@bermuda.ucd.ie \\ 2 Max-Planck-Institut für extraterrestrische Physik, 85748 Garching, Germany
}

Received 2 August 2007 / Accepted 4 March 2008

\section{ABSTRACT}

\begin{abstract}
Context. INTEGRAL has two sensitive gamma-ray instruments that have detected and localised 47 gamma-ray bursts (GRBs) from its launch in October 2002 up to July 2007.

Aims. We present the spectral, spatial, and temporal properties of the bursts in the INTEGRAL GRB catalogue using data from the imager, IBIS, and spectrometer, SPI.

Methods. Spectral properties of the GRBs are determined using power-law and, where appropriate, Band model and quasithermal model fits to the prompt emission. Spectral lags, i.e. the time delay in the arrival of low-energy $\gamma$-rays with respect to high-energy $\gamma$-rays, are measured for 31 of the GRBs.

Results. The photon index distribution of power-law fits to the prompt emission spectra is presented and is consistent with that obtained by Swift. The peak flux distribution shows that INTEGRAL detects proportionally more weak GRBs than Swift because of its higher sensitivity in a smaller field of view. The all-sky rate of GRBs above $\sim 0.15 \mathrm{ph} \mathrm{cm}^{-2} \mathrm{~s}^{-1}$ is $\sim 1400 \mathrm{yr}^{-1}$ in the fully coded field of view of IBIS. Two groups are identified in the spectral lag distribution of INTEGRAL GRBs, one with short lags $<0.75 \mathrm{~s}$ (between 25-50 keV and 50-300 keV) and one with long lags $>0.75 \mathrm{~s}$. Most of the long-lag GRBs are inferred to have low redshifts because of their long spectral lags, their tendency to have low peak energies, and their faint optical and X-ray afterglows. They are mainly observed in the direction of the supergalactic plane with a quadrupole moment of $Q=-0.225 \pm 0.090$ and hence reflect the local large-scale structure of the Universe.

Conclusions. The spectral, spatial, and temporal properties of the 47 GRBs in the INTEGRAL catalogue are presented and compared with the results from other missions. The rate of long-lag GRBs with inferred low luminosity is $\sim 25 \%$ of type Ib/c supernovae. Some of these bursts could be produced by the collapse of a massive star without a supernova. Alternatively, they could result from a different progenitor, such as the merger of two white dwarfs or a white dwarf with a neutron star or black hole, possibly in the cluster environment without a host galaxy.
\end{abstract}

Key words. gamma rays: bursts - gamma rays: observations

\section{Introduction}

The prompt emission of gamma-ray bursts provides valuable insight into the mechanisms from which these extremely explosive events originate. Their short durations and highly variable temporal structures provide constraints on the physics of the central engine powering the burst. A number of GRB reviews have been published (e.g. Zhang \& Mészáros 2004; Piran 2005; Mészáros 2006). In recent years, the advent of missions such as the Compton Gamma-Ray Observatory (CGRO) (Fishman \& Meegan 1995), along with the improved imaging capabilities of missions such as BeppoSAX (Boella et al. 1997), HETE II (Sakamoto et al. 2005), INTEGRAL (Winkler et al. 2003), and Swift (Gehrels et al. 2004), has led to the precise localisations of GRBs and enabled rapid multi-wavelength follow-up observations. The $\mathrm{X}$-ray, optical and radio afterglow detections are

\footnotetext{
* Based on observations with INTEGRAL, an ESA project with instruments and science data centre funded by ESA member states (especially the PI countries: Denmark, France, Germany, Italy, Switzerland, Spain), Czech Republic and Poland, and with the participation of Russia and the USA.

$\star \star$ Appendix A is only available in electronic form at http://www . aanda.org
}

Table 1. Afterglow detections for GRBs localised by recent $\gamma$-ray missions between July 1996 and July 2007.

\begin{tabular}{lcccc}
\hline \hline & BeppoSAX & HETE II & INTEGRAL & Swift \\
\hline GRBs & 55 & 79 & $\mathbf{4 7}$ & 242 \\
X-ray & 31 & 19 & $\mathbf{1 7}$ & 209 \\
Optical & 17 & 30 & $\mathbf{1 6}$ & 123 \\
Radio & 11 & 8 & $\mathbf{8}$ & 17 \\
\hline
\end{tabular}

listed in Table 1 for a total of 423 GRBs well localised by these missions between July 1996 and July 2007, showing in particular the observed number of afterglows based on INTEGRAL GRB detections. The data are taken from the webpage maintained by Jochen Greiner ${ }^{1}$.

There are two main $\gamma$-ray instruments on board INTEGRAL, namely IBIS (Ubertini et al. 2003) and SPI (Vedrenne et al. 2003), optimised for high-resolution imaging and spectroscopy of the $\gamma$-ray sky, respectively. The IBIS instrument is comprised of two separate layers of dectectors, ISGRI in the $15 \mathrm{keV}-1 \mathrm{MeV}$ energy range (Lebrun et al. 2003), and PICsIT in the $180 \mathrm{keV}-10 \mathrm{MeV}$ energy range (Labanti et al. 2003).

\footnotetext{
${ }^{1}$ http://www .mpe.mpg.de/ jcg/grbgen.html
} 
IBIS/ISGRI has 16384 CdTe detectors, located $3.4 \mathrm{~m}$ from a tungsten mask which projects a shadowgram on the detector plane. Maps of the sky are reconstructed by decoding the shadowgram with the mask pattern. IBIS has a fully coded field of view $(\mathrm{FCFOV})$ of $9^{\circ} \times 9^{\circ}$ and a partially coded field of view $(\mathrm{PCFOV})$ of $19^{\circ} \times 19^{\circ}$ at $50 \%$ coding and $29^{\circ} \times 29^{\circ}$ at zero coding. SPI consists of 19 hexagonal germanium $(\mathrm{Ge})$ detectors covering the energy range $20 \mathrm{keV}-8 \mathrm{MeV}$ with high energy resolution of $2.5 \mathrm{keV}$ at $1.3 \mathrm{MeV}$. A coded mask is located $1.71 \mathrm{~m}$ above the detector plane for imaging purposes, giving a $16^{\circ}$ corner-to-corner FCFoV and a PCFoV of $34^{\circ}$. The SPI and IBIS instruments are supported by an optical camera (OMC, Mas-Hesse et al. 2003) and an X-ray monitor (JEM-X, Lund et al. 2003).

The INTEGRAL Burst Alert System (IBAS ${ }^{2}$, Mereghetti et al. 2003b) is an automatic ground-based system for the accurate localisation of GRBs and the rapid distribution of GRB coordinates, providing, on average, $0.8 \mathrm{GRBs}$ per month with an error radius of $\sim 3$ arcmin. INTEGRAL has detected 46 longduration GRBs $\left(T_{90} \gtrsim 2 \mathrm{~s}\right)$ and 1 short-duration GRB $\left(T_{90} \lesssim\right.$ 2 s) between October 2002 and July 2007. INTEGRAL bursts of particular interest include the low-luminosity GRB 031203 (Sazonov et al. 2004), the very intense GRB 041219a (McBreen et al. 2006), a number of X-ray rich GRBs such as GRB 040223 (McGlynn et al. 2005; Filliatre et al. 2006), GRB 040403 (Mereghetti et al. 2005) and GRB 040624 (Filliatre et al. 2006), and the short-duration GRB 070707 (McGlynn et al. 2008a). In addition, Marcinkowski et al. (2006) have detected a bright, hard GRB outside the field of view using the ISGRI Compton mode. Spectroscopic redshifts have been determined for four INTEGRAL GRBs, i.e. GRB 031203 at $z=0.1055$ (Prochaska et al. 2004); GRB 050223 at $z=0.584$ (Pellizza et al. 2006); GRB 050525a at $z=0.606$ (Foley et al. 2005) and GRB 050502a at $z=3.793$ (Prochaska et al. 2005). Non-spectroscopic redshifts have been inferred for GRB $040812(0.3<z<0.7$, D'Avanzo et al. 2006) and GRB $040827(0.5<z<1.7$, de Luca et al. 2005). The low efficiency for measuring redshifts is partially due to the fact that INTEGRAL spends a large amount of observing time pointing towards the galactic plane.

Gamma-ray burst continuum spectra are in most cases well described by a smoothly broken power law in the $30 \mathrm{keV}-2 \mathrm{MeV}$ energy range (Band et al. 1993). The $\gamma$-ray spectral shape as predicted for optically thin synchrotron emission are two asymptotically broken power laws but many GRBs are not consistent with this model and it requires modification to fit the observed spectra (e.g. Lloyd-Ronning \& Petrosian 2002). It has been proposed that GRB spectra may contain a thermal component (e.g. Ghirlanda et al. 2003; Ryde 2005; Kaneko et al. 2006; McBreen et al. 2006). The interpretation of quasithermal emission as opposed to synchrotron emission can provide an explanation for the observed spectral characteristics within a more physical framework (e.g. Rees \& Mészáros 2005; Ryde et al. 2006).

The time profiles of GRBs often exhibit a complex and unpredictable nature, displaying considerable diversity both in terms of structure and duration (McBreen et al. 2001; Quilligan et al. 2002). This makes them difficult to classify on the basis of temporal structure alone. One notable feature of GRB time profiles is the tendency for emission in a high-energy band to lead the arrival of photons in a low-energy band (e.g. Cheng et al. 1995; Wu \& Fenimore 2000; Norris et al. 2000; Bolmont et al. 2006; McBreen et al. 2006; Hakkila et al. 2007, 2008). The

\footnotetext{
${ }^{2}$ http://ibas.iasf-milano.inaf.it/IBAS_Results.html
}

energy-dependent lag allows the temporal and spectral properties of the GRB prompt $\gamma$-ray emission to be combined in a single measurement. The typical lag values measured for longduration GRBs detected by the Burst and Transient Source Experiment (BATSE) between the $25-50 \mathrm{keV}$ and $100-300 \mathrm{keV}$ channels concentrate $\sim 100 \mathrm{~ms}$ (Norris et al. 2000). An anticorrelation between spectral lag and isotropic peak luminosity was first observed by Norris et al. (2000), using 6 BATSE bursts with measured redshifts. A similar trend is observed between lag and luminosity for a number of Swift GRBs of known redshift (Gehrels et al. 2006). However, there exist notable outliers, in particular the ultra-low luminosity bursts GRB 980425, GRB 031203 and GRB 060218, associated with the supernovae SN 1998bw, SN 20031w and SN 2006aj, respectively. Short bursts $\left(T_{90}<2 \mathrm{~s}\right)$ have very small or negligible lags (Yi et al. 2006; Norris \& Bonnell 2006; Zhang et al. 2006a) and relatively low peak luminosities and so do not lie on the correlation (Gehrels et al. 2006). On this basis, the spectral lag has been suggested by Donaghy et al. (2006) as one of the criteria to determine whether a burst is long or short. Pulse width and spectral lag are strongly related, with wider pulses tending to have longer spectral lags (Norris \& Bonnell 2006). Relative spectral lags, defined as the ratio of spectral lag to pulse width, have been found to have normal distributions centering on $\sim 100 \mathrm{~ms}$ for long bursts (Zhang et al. 2006b) and $\sim 14 \mathrm{~ms}$ for short GRBs (Zhang et al. 2006a). The lag-luminosity and $E_{\text {peak }}-E_{\text {iso }}$ (Amati et al. 2007) relationships can be used as distance indicators for GRBs (Schaefer 2007), provided the role of selection effects is understood and quantified (e.g. Butler et al. 2008).

The physical basis underlying spectral lags is not yet well understood (Schaefer 2004). The observed lag of a burst is a direct consequence of its spectral evolution because the peak of the $v F_{v}$ spectrum, $E_{\text {peak }}$, decays with time (Kocevski \& Liang 2003; Hafizi \& Mochkovitch 2007). The internal shock model allows for three possible sources of temporal variations in GRB pulses: cooling, hydrodynamics and geometric angular effects. Cooling is unable to fully account for the time lag since the synchrotron timescale is much shorter than the lag timescale (Wu \& Fenimore 2000). It has been proposed that the lag-luminosity relation may arise kinematically, based on the viewing angle at which the GRB jet is observed (Salmonson 2000). In this interpretation, a high-luminosity GRB with short spectral lag corresponds to a jet with a small viewing angle, while a low-luminosity GRB with long spectral lag corresponds to a jet with a large viewing angle (Ioka \& Nakamura 2001). A correlation has also been observed between spectral lag (or luminosity) and jet-break time, thereby connecting the prompt and afterglow phases of GRBs. This may be understood in terms of a model in which the Lorentz factor decreases away from the axis of the GRB jet (Salmonson \& Galama 2002). The connection between spectral lag and the timescales involved in the hydrodynamic processes and radiative mechanisms of the burst has been discussed by Daigne \& Mochkovitch (2003).

A subpopulation of local, faint, long-lag GRBs has been suggested by Norris (2002) from a study of BATSE bursts, which implies that events with low peak fluxes $\left(F_{\text {Peak }}(50-300 \mathrm{keV})\right.$ $\sim 0.25 \mathrm{ph} \mathrm{cm}^{-2} \mathrm{~s}^{-1}$ ) should be predominantly long-lag GRBs. The sensitivity of IBIS is such that bursts fainter than the BATSE limit can be well localised. In this paper we present the spectral, spatial and temporal lag properties of the complete sample of the 47 GRBs detected in the field of view of IBIS and SPI up to July 2007. Section 2 describes the capabilities of SPI's anticoincidence shield as a GRB detector. The spectral and lag analyses are described in Sect. 3 and results are presented in Sect. 4. 

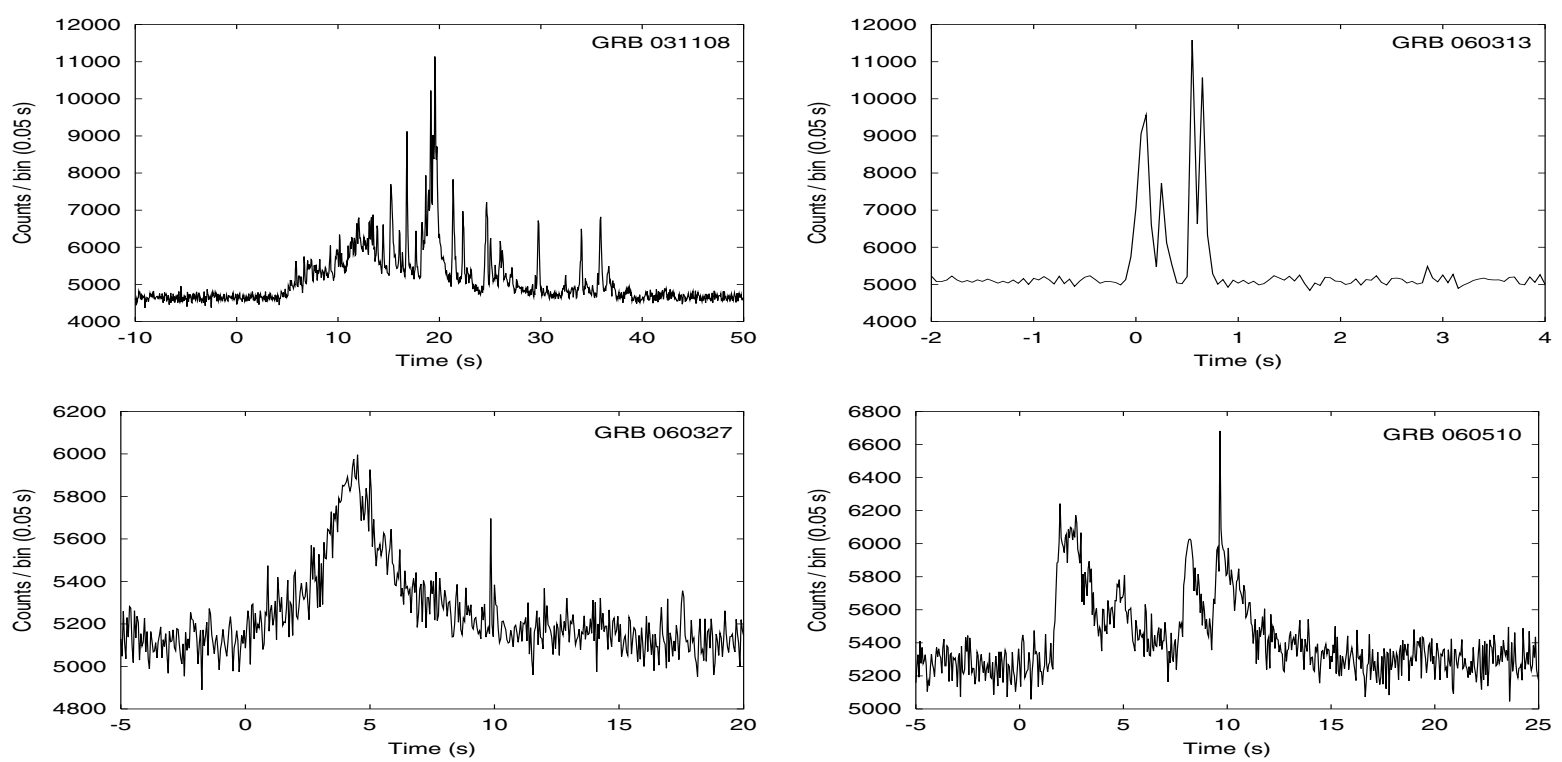

Fig. 1. A selection of GRB lightcurves detected by the Anti-Coincidence Shield at photon energies $>80 \mathrm{keV}$.

Sections 5 and 6 discuss the significance of these results, which imply a large population of long-lag GRBs with low luminosities. The cosmological parameters adopted throughout the paper are $H_{0}=70 \mathrm{~km} \mathrm{~s}^{-1} \mathrm{Mpc}^{-1}, \Omega_{\mathrm{m}}=0.3, \Omega_{\mathrm{vac}}=0.7$. All errors are quoted at the $1 \sigma$ confidence level.

\section{GRBs detected with SPI's Anti-Coincidence Shield}

In addition to SPI and IBIS, the Anti-Coincidence Shield (ACS) surrounding the SPI detectors works as a highly-sensitive GRB detector above $\sim 80 \mathrm{keV}$ but lacks spatial and spectral information (von Kienlin et al. 2003b). The ACS consists of 91 BGO crystals with a total mass of $512 \mathrm{~kg}$ surrounding SPI. It has a maximum sensitivity to GRBs at $\sim 90^{\circ}$ from the pointing direction and provides lightcurves in $50 \mathrm{~ms}$ intervals. The ACS detects GRBs at a rate of $\sim 1$ every 2-3 days. A selection of GRBs detected with SPI-ACS is shown in Fig. 1 using data taken from the publicly available catalogue of SPI-ACS GRBs ${ }^{3}$. Temporal analysis of a more complete sample is presented in Rau et al. (2005). The ACS is used as part of the interplanetary network (Hurley et al. 2006).

\section{Analysis of GRBs observed with IBIS and SPI}

\subsection{Spectral analysis}

Spectral analysis of the GRBs was performed using INTEGRAL's Online Software Analysis version 5.1 available from the INTEGRAL Science Data Centre ${ }^{4}$. The $T_{90}$ duration (i.e., the time during which $5 \%$ to $95 \%$ of the GRB counts are recorded) was determined for each GRB in the 20-200 keV energy range and the spectrum was generated for that time interval. For cases in which the GRB had a multi-peaked time profile, the GRB was divided into its constituent pulses and a spectrum was generated for each pulse to investigate evolution of the spectral parameters during the burst.

\footnotetext{
${ }_{3}$ http://wWw .mpe.mpg.de/gamma/science/grb/1ACSburst. html

${ }^{4}$ http://isdc.unige.ch/
}

Each GRB was fit by a simple power-law model, the Band model and a quasithermal (combined power-law + blackbody) model. Weak GRBs are best fit by a single power-law model because the limited statistics are insufficient to constrain any additional parameters. For brighter GRBs, the Band model or combined power-law + blackbody fits usually result in an improved $\chi^{2}$ value. The peak flux was measured over the brightest $1 \mathrm{~s}$ time interval in the $20-200 \mathrm{keV}$ energy range. The fluence of each GRB was determined for the $T_{90}$ interval in the $20-200 \mathrm{keV}$ energy range for the IBIS spectra, and the $20-200 \mathrm{keV}$ and $20 \mathrm{keV}-8 \mathrm{MeV}$ energy ranges for the SPI spectra.

\subsection{Spectral lag analysis}

In order to measure the lag, background-subtracted lightcurves were extracted in three energy bands comparable to those used with BATSE, namely 25-50 keV (Channel 1), 50-100 keV (Channel 2) and 100-300 keV (Channel 3). The lag, $\tau$, between two energy channels was determined by computing the crosscorrelation function $(\mathrm{CCF})$ between the two lightcurves as a function of temporal lag as described by Band (1997) and Norris et al. (2000). Assuming the time profiles in both energy channels display sufficient similarity, the peak in the CCF then corresponds to the time lag of the GRB between the two energy channels in question. The lag was determined between Channels 1 and 2, $\tau_{2,1}$, Channels 1 and $3, \tau_{3,1}$, and to account for those cases in which the signal level in Channel 3 was insufficient to determine an accurate lag, the counts in Channels 2 and 3 were combined and correlated with Channel 1 to give $\tau_{2+3,1}$. In this paper, GRBs with $\tau_{2+3,1}>0.75 \mathrm{~s}$ are defined as long-lag.

The reliability of the cross-correlation technique was limited primarily by the signal to noise ratio of the data. The faint nature of many of the GRBs detected by INTEGRAL can result in a noisy $\mathrm{CCF}$ with an ambiguous peak. A denoising technique was used to smooth the lightcurve while retaining the structure of the burst. This technique involved filtering the signal to remove the high frequency components in order to produce a denoised lightcurve (Quilligan et al. 2002). The wavelet analysis was carried out on the weakest GRBs with $F_{\text {peak }}(20-200 \mathrm{keV}) \lesssim$ $0.6 \mathrm{ph} \mathrm{cm}^{-2} \mathrm{~s}^{-1}$ using the wavelet toolbox in MATLAB. As an example, the lightcurves of GRB 070615 in the $25-50 \mathrm{keV}$ 

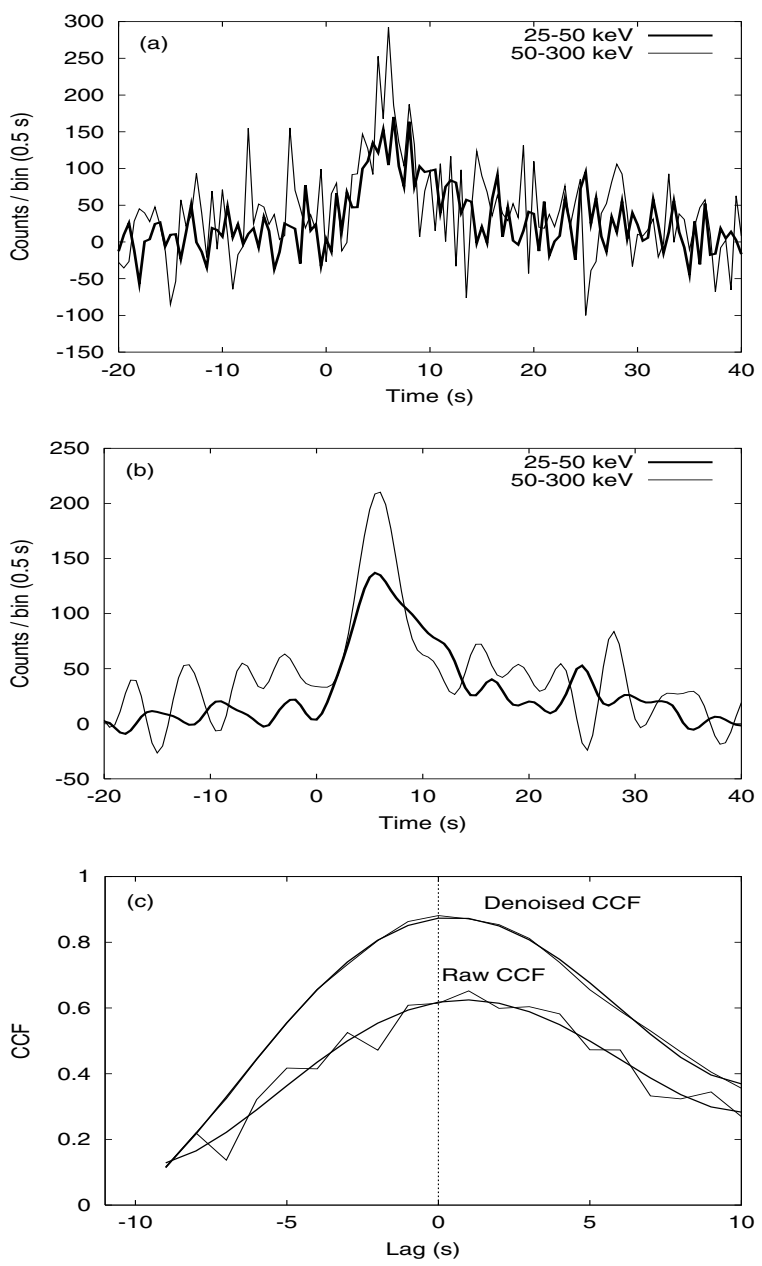

Fig. 2. a) Raw and b) denoised lightcurves of GRB 070615 in the 25-50 keV (dark line) and 50-300 keV (light line) energy bands. c) Cross correlation functions and polynomial fits giving a lag of $0.40_{-0.25}^{+0.15}$ and $0.40_{-0.20}^{+0.15}$ for the raw and denoised lightcurve data, respectively. The dashed line represents a lag of $0 \mathrm{~s}$.

and 50-300 keV energy bands are shown in Fig. 2a before and Fig. $2 b$ after denoising. The CCFs resulting from the raw data and denoised data are plotted in Fig. 2c. The denoised data results in a smoother CCF peak and a more significant correlation, while retaining the position of the peak at a similar lag value to that measured with the raw data. Six INTEGRAL GRBs were too weak for a reliable lag to be determined using either the raw or denoised data.

The CCF was fit with a fourth order polynomial in order to account for the asymmetry of the CCF (Norris et al. 2000). The peak of the polynomial fit to the CCF was then taken to be the true lag value. For each GRB, an average spectral lag over the total burst duration was determined. For the cases in which separate pulses could be clearly distinguished by eye, spectral lags were measured for the individual pulses to investigate the evolution of spectral lag during the burst. In each case, the spectral lag was determined for regions of the lightcurve above $10 \%, 30 \%$ and $50 \%$ of the peak count rate and over a number of different lag ranges to ensure that consistent results were obtained. The optimum lag range was taken to be that for which the CCF was concave down but short enough that the CCF peak was well fit. Statistical errors were calculated using a bootstrap method as described in Norris et al. (2000). This involves adding Poissonian noise based on the observed counts to the lightcurves in the
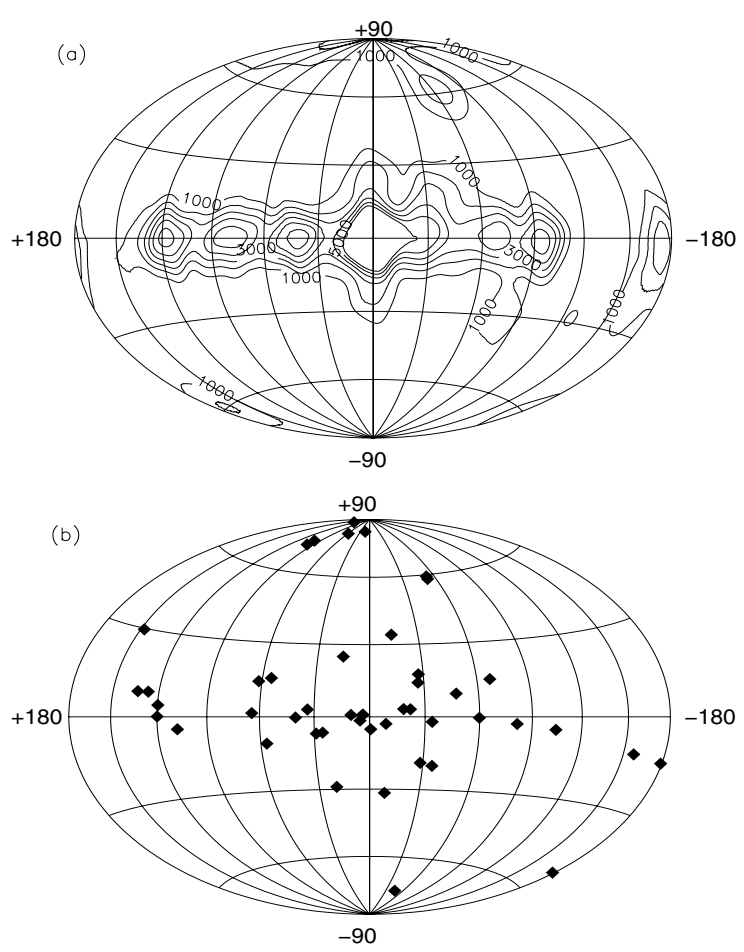

Fig. 3. a) INTEGRAL exposure map in galactic coordinates from October 2002 up to July 2007 (contours in units of kiloseconds), showing the concentration of exposure in the direction of the galactic plane (Erik Kuulkers, private communication). b) Spatial distribution of 47 INTEGRAL GRBs detected between October 2002 and July 2007 in galactic coordinates.

different energy channels and re-computing the CCF in 100 realisations for each burst. The 50th ranked value is then the mean lag and the 16th and 84th ranked values represent $\pm 1 \sigma$. The lightcurve data was over-resolved by a factor of 10 in order to compute the errors at a time resolution less than the natural binning of the raw data.

\section{Results}

The exposure map and spatial distribution of the 47 GRBs observed with IBIS are shown in galactic coordinates in Fig. 3 for the period from October 2002 to July 2007. The burst distribution is significantly concentrated towards the galactic plane, reflecting the direction in which the satellite is pointed. INTEGRAL spent $64 \%$ of its observing time in the half of the sky at galactic latitudes between $\pm 30^{\circ}$.

The properties of the 47 INTEGRAL GRBs are presented in Tables 2 and 3 . The coordinates and afterglow information are taken from the webpage maintained by Jochen Greiner. The typical size of the INTEGRAL error box is $3^{\prime}$. GRB $060428 \mathrm{c}$ did not trigger IBAS but was discovered during subsequent analysis of INTEGRAL archival data (Grebenev \& Chelovekov 2007). The spectral lag for each burst is presented in Table 4, with 11 GRBs having long lags $\left(\tau_{2+3,1}>0.75 \mathrm{~s}\right)$. The off-axis angle distribution of the GRBs in the IBIS FoV is given in Fig. 4. The bursts have preferentially higher peak fluxes at larger angles outside the FCFoV of IBIS due to the reduced sensitivity at lower coding levels. The $\log N-\log P$ distribution is given in Fig. 5 for all IBIS GRBs and separately for the small subsample of 11 longlag GRBs. The long-lag GRBs appear to form a separate population at low values of $P$. The $T_{90}$ distribution of INTEGRAL 
Table 2. GRBs detected by INTEGRAL. The columns refer to (from left to right): GRB; right ascension; declination; $T_{90}$ duration; peak flux; fluence; photon index; $\chi^{2}$ /degrees of freedom (d.o.f.); afterglow detections in radio, $\mathrm{R}$, infrared, IR, optical, O and X-ray, X. Peak fluxes, fluences and photon indices are given in the $20-200 \mathrm{keV}$ energy range.

\begin{tabular}{|c|c|c|c|c|c|c|c|c|c|}
\hline \multirow[t]{2}{*}{$\overline{\mathrm{GRB}}$} & \multirow[t]{2}{*}{ RA } & \multirow[t]{2}{*}{ Dec } & \multirow{2}{*}{$\begin{array}{r}T_{90} \\
\mathrm{~S}\end{array}$} & \multicolumn{2}{|c|}{ Peak flux } & \multirow{2}{*}{$\begin{array}{l}\text { Fluence } \\
\mathrm{erg} \mathrm{cm}^{-2}\end{array}$} & \multirow[t]{2}{*}{ Photon index } & \multirow{2}{*}{$\begin{array}{c}\chi^{2} \\
\text { /d.o.f. }\end{array}$} & \multirow[t]{2}{*}{ Afterglow } \\
\hline & & & & $\mathrm{ph} \mathrm{cm}^{-2} \mathrm{~s}^{-1}$ & 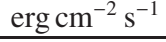 & & & & \\
\hline GRB $021125^{1}$ & $19: 47: 57$ & $28: 23: 35$ & 24 & 22 & - & $4.8 \times 10^{-5^{*}}$ & -2.2 & - & - \\
\hline GRB $021219^{2}$ & $18: 50: 27$ & $31: 57: 17$ & 5.5 & 3.7 & $3.5 \times 10^{-7}$ & $9.0 \times 10^{-7}$ & $-2.00 \pm 0.10$ & 一 & $\mathrm{R}$ \\
\hline GRB $030131^{3}$ & $13: 28: 21$ & $30: 40: 43$ & 124 & 1.9 & $1.7 \times 10^{-7}$ & $7.0 \times 10^{-6}$ & Table 3 & 一 & $\mathrm{O}$ \\
\hline GRB $030227^{4}$ & $04: 57: 34$ & $20: 28: 16$ & 33 & 1.1 & $9.6 \times 10^{-8}$ & $7.5 \times 10^{-7}$ & $-1.85 \pm 0.20$ & - & $\mathrm{X}, \mathrm{O}$ \\
\hline GRB $030320^{5}$ & $17: 51: 36$ & $-25: 18: 52$ & 48 & 5.7 & $5.4 \times 10^{-7}$ & $1.1 \times 10^{-5}$ & $-1.69 \pm 0.08$ & 一 & - \\
\hline GRB $030501^{6}$ & $19: 05: 33$ & $06: 15: 57$ & 40 & 2.7 & $2.5 \times 10^{-7}$ & $3.0 \times 10^{-6}$ & $-1.75 \pm 0.10$ & 一 & - \\
\hline GRB 030529 & 09:40:30 & $-56: 20: 31$ & 20 & 0.4 & $3.7 \times 10^{-8}$ & $4.0 \times 10^{-7}$ & $-2.07 \pm 0.30$ & $2.9 / 5$ & - \\
\hline GRB $031203^{7 \star}$ & 08:02:32 & $-39: 50: 47$ & 39 & 2.6 & $2.5 \times 10^{-7}$ & $2.0 \times 10^{-6}$ & $-1.63 \pm 0.06$ & - & $\mathrm{X}, \mathrm{O}, \mathrm{R}$ \\
\hline GRB $040106^{8}$ & $11: 52: 18$ & $-46: 47: 15$ & 47 & 0.6 & $6.5 \times 10^{-8}$ & $8.2 \times 10^{-7}$ & $-1.72 \pm 0.15$ & 一 & $\mathrm{X}, \mathrm{O}, \mathrm{R}$ \\
\hline GRB $040223^{9}$ & $16: 39: 31$ & $-41: 55: 47$ & 258 & 0.2 & $1.6 \times 10^{-8}$ & $4.4 \times 10^{-7}$ & $-2.30 \pm 0.19$ & $20.1 / 20$ & $x$ \\
\hline GRB 040323 & $13: 53: 49$ & $-52: 20: 45$ & 14 & 1.5 & $2.0 \times 10^{-7}$ & $3.0 \times 10^{-6}$ & $-1.44 \pm 0.18$ & $10.6 / 8$ & $\mathrm{O}$ \\
\hline GRB $040403^{10}$ & $07: 40: 54$ & $68: 12: 55$ & 21 & 0.5 & $4.3 \times 10^{-8}$ & $5.0 \times 10^{-7}$ & $-1.90 \pm 0.15$ & - & 一 \\
\hline GRB $040422^{11}$ & $18: 42: 01$ & 01:59:04 & 4 & 2.3 & $1.8 \times 10^{-7}$ & $3.4 \times 10^{-7}$ & Table 3 & 一 & IR \\
\hline GRB $040624^{12}$ & 13:00:08 & $-03: 34: 08$ & 62 & 0.7 & $4.0 \times 10^{-8}$ & $7.0 \times 10^{-6}$ & $-2.11 \pm 0.20$ & $28.1 / 33$ & - \\
\hline GRB 040730 & $15: 53: 14$ & $-56: 28: 15$ & 51 & 0.32 & $3.4 \times 10^{-8}$ & $6.6 \times 10^{-7}$ & $-1.44 \pm 0.14$ & $34.3 / 33$ & - \\
\hline GRB 040812 & $16: 26: 05$ & $-44: 42: 32$ & 16 & 0.7 & $5.0 \times 10^{-8}$ & $2.3 \times 10^{-7}$ & $-2.34 \pm 0.29$ & $19.5 / 26$ & $\mathrm{X}, \mathrm{R}$ \\
\hline GRB 040827 & $15: 17: 00$ & $-16: 08: 21$ & 35 & 0.42 & $6.0 \times 10^{-8}$ & $1.2 \times 10^{-6}$ & $-1.83 \pm 0.18$ & $32.5 / 29$ & $\mathrm{X}, \mathrm{O}$ \\
\hline XRF 040903 & $18: 03: 22$ & $-25: 15: 23$ & 15 & 0.23 & $2.0 \times 10^{-8}$ & $1.4 \times 10^{-7}$ & $-2.94 \pm 0.44$ & $16.6 / 20$ & - \\
\hline GRB 041015 & $00: 18: 37$ & $66: 51: 37$ & 33 & 0.17 & $4.0 \times 10^{-8}$ & $6.0 \times 10^{-7}$ & $-0.95 \pm 0.28$ & $17.4 / 21$ & - \\
\hline GRB 041218 & 01:39:06 & $71: 20: 05$ & 40 & 3.19 & $2.8 \times 10^{-7}$ & $5.5 \times 10^{-6}$ & Table 3 & - & $\mathrm{O}$ \\
\hline GRB 041219a ${ }^{13}$ & $00: 24: 26$ & $62: 50: 06$ & 186 & 33 & $3.6 \times 10^{-6}$ & $1.6 \times 10^{-4}$ & Table 3 & 一 & $\mathrm{O}, \mathrm{R}$ \\
\hline GRB 050129 & $16: 51: 12$ & $-03: 04: 44$ & 40 & 0.36 & $3.0 \times 10^{-8}$ & $4.5 \times 10^{-7}$ & $-1.91 \pm 0.31$ & $21.6 / 26$ & - \\
\hline GRB 050223* & $18: 05: 36$ & $-62: 28: 26$ & 39 & 0.67 & $6.0 \times 10^{-8}$ & $8.2 \times 10^{-7}$ & $-1.84 \pm 0.25$ & $21.3 / 30$ & $X$ \\
\hline GRB $050502 a^{\star}$ & $13: 29: 45$ & 42:40:27 & 20 & 1.58 & $2.0 \times 10^{-7}$ & $>1.4 \times 10^{-6}$ & Table 3 & - & $\mathrm{O}, \mathrm{IR}$ \\
\hline GRB 050504 & $13: 24: 00$ & $40: 41: 45$ & 58 & 0.45 & $7.4 \times 10^{-8}$ & $1.3 \times 10^{-6}$ & $-1.32 \pm 0.10$ & $50.8 / 29$ & $X$ \\
\hline GRB 050520 & $12: 50: 03$ & $30: 27: 02$ & 80 & 0.53 & $4.0 \times 10^{-8}$ & $2.4 \times 10^{-6}$ & $-1.61 \pm 0.07$ & $26.1 / 30$ & $\mathrm{X}, \mathrm{R}$ \\
\hline XRF 050522 & $13: 20: 35$ & $24: 47: 30$ & 17 & 0.2 & $1.3 \times 10^{-8}$ & $7.0 \times 10^{-8}$ & $-2.72 \pm 0.67$ & $16.2 / 17$ & - \\
\hline GRB $050525 a^{\star}$ & $18: 32: 33$ & $26: 20: 23$ & 12 & 31.7 & $3.1 \times 10^{-6}$ & $2.0 \times 10^{-5}$ & Table 3 & - & $\mathrm{O}, \mathrm{R}$ \\
\hline GRB 050626 & $12: 26: 58$ & $-63: 08: 03$ & 50 & 0.27 & $2.0 \times 10^{-8}$ & $7.6 \times 10^{-7}$ & $-2.20 \pm 0.14$ & $27.6 / 30$ & - \\
\hline GRB 050714a & $02: 54: 21$ & 69:07:34 & 34 & 0.18 & $1.5 \times 10^{-8}$ & $4.7 \times 10^{-7}$ & $-2.06 \pm 0.20$ & $21.8 / 28$ & - \\
\hline GRB 050918 & $17: 50: 26$ & $-25: 24: 51$ & 115 & 1.61 & $1.6 \times 10^{-7}$ & $3.1 \times 10^{-6}$ & Table 3 & - & - \\
\hline GRB 050922a & 18:04:37 & $-32: 01: 24$ & 8 & 0.01 & $5.3 \times 10^{-9}$ & $5.3 \times 10^{-8}$ & $-1.85 \pm 1.01$ & $0.6 / 2$ & - \\
\hline GRB 051105b & $00: 37: 51$ & $-40: 28: 52$ & 16 & 0.31 & $3.0 \times 10^{-8}$ & $2.9 \times 10^{-7}$ & $-1.83 \pm 0.23$ & $25.7 / 27$ & - \\
\hline GRB 051211b & $23: 02: 45$ & $55: 04: 44$ & 60 & 0.6 & $6.1 \times 10^{-8}$ & $2.0 \times 10^{-6}$ & $-1.62 \pm 0.10$ & $38.4 / 28$ & $\mathrm{X}, \mathrm{R}$ \\
\hline GRB 060114 & 13:01:07 & $-04: 44: 53$ & 63 & 0.31 & $3.0 \times 10^{-8}$ & $9.0 \times 10^{-7}$ & $-0.98 \pm 0.16$ & $18.9 / 30$ & - \\
\hline GRB 060130 & $15: 16: 54$ & $-36: 54: 43$ & 29 & 0.11 & $7.7 \times 10^{-9}$ & $2.8 \times 10^{-7}$ & $-1.51 \pm 0.40$ & $27.7 / 22$ & - \\
\hline GRB 060204a & $15: 28: 56$ & $-39: 26: 38$ & 34 & 0.16 & $2.1 \times 10^{-8}$ & $6.6 \times 10^{-7}$ & $-1.43 \pm 0.27$ & $20.3 / 26$ & $X$ \\
\hline GRB $060428 c^{14}$ & 19:00:52 & $-09: 33: 00$ & 12 & 3.9 & $3.6 \times 10^{-7}$ & $2.3 \times 10^{-6}$ & Table 3 & - & - \\
\hline GRB $060901^{15}$ & $19: 08: 38$ & $-06: 38: 22$ & 20 & 8.6 & $9.0 \times 10^{-7}$ & $8.7 \times 10^{-6}$ & Table 3 & - & $\mathrm{X}, \mathrm{O}$ \\
\hline GRB 060912b & $18: 04: 52$ & $-19: 52: 50$ & 91 & 0.08 & $8.8 \times 10^{-9}$ & $7.1 \times 10^{-7}$ & $-1.52 \pm 0.23$ & $18.5 / 33$ & - \\
\hline GRB $060930^{\dagger}$ & 20:18:09 & $-23: 37: 31$ & 20 & 0.3 & $2.2 \times 10^{-8}$ & $2.5 \times 10^{-7}$ & - & - & - \\
\hline GRB 061025 & $20: 03: 39$ & $-48: 14: 39$ & 11 & 1.14 & $1.3 \times 10^{-7}$ & $1.1 \times 10^{-6}$ & Table 3 & - & $\mathrm{X}, \mathrm{O}$ \\
\hline GRB 061122 & $20: 15: 21$ & $15: 30: 51$ & 11 & 31.7 & $3.1 \times 10^{-6}$ & $2.0 \times 10^{-5}$ & Table 3 & - & $\mathrm{X}, \mathrm{O}$ \\
\hline GRB 070309 & $17: 34: 44$ & $-37: 56: 40$ & 38 & 0.13 & $1.1 \times 10^{-8}$ & $5.4 \times 10^{-7}$ & $-1.73 \pm 0.27$ & $6.5 / 26$ & $X ?$ \\
\hline GRB $070311^{\dagger}$ & $05: 50: 10$ & 03:22:29 & 50 & 0.9 & - & $2.0 \times 10^{-6}$ & - & - & $\mathrm{X}, \mathrm{O}$ \\
\hline GRB 070615 & $02: 57: 14$ & $-04: 24: 28$ & 27 & 0.41 & $2.8 \times 10^{-8}$ & $5.0 \times 10^{-7}$ & $-1.62 \pm 0.28$ & $21.9 / 27$ & - \\
\hline GRB 070707 & 17:51:00 & $-68: 52: 52$ & 0.8 & 1.72 & $2.1 \times 10^{-7}$ & $2.1 \times 10^{-7}$ & $-1.19 \pm 0.14$ & $51.2 / 47$ & $\mathrm{X}, \mathrm{O}$ \\
\hline
\end{tabular}

${ }^{1}$ Malaguti et al. (2003), ${ }^{2}$ Mereghetti et al. (2003a), ${ }^{3}$ Götz et al. (2003), ${ }^{4}$ Mereghetti et al. (2003c), ${ }^{5}$ von Kienlin et al. (2003a), ${ }^{6}$ Beckmann et al. (2003), ${ }^{7}$ Sazonov et al. (2004), ${ }^{8}$ Moran et al. (2005), ${ }^{9}$ McGlynn et al. (2005), ${ }^{10}$ Mereghetti et al. (2005), ${ }^{11}$ Filliatre et al. (2005), ${ }^{12}$ Filliatre et al. (2006), ${ }^{13}$ McBreen et al. (2006), ${ }^{14}$ Grebenev \& Chelovekov (2007), ${ }^{15}$ A. von Kienlin (priv. comm.).

* 25-500 keV.

* GRBs with known redshift; GRB 031203 at $z=0.106$ (Prochaska et al. 2004); GRB 050223 at $z=0.584$ (Pellizza et al. 2006); GRB 050502a at $z=3.793$ (Prochaska et al. 2005); GRB 050525a (Foley et al. 2005) at $z=0.606$.

$\lceil$ Data unavailable for GRB 060930 (values taken from Gotz et al. 2006) and GRB 070311 (values taken from Mereghetti et al. 2007).

GRBs is shown in Fig. 6 and compared with the bimodal distribution for BATSE GRBs (Kouveliotou et al. 1993). There is reasonable agreement between the two distributions, especially when the small number of INTEGRAL GRBs is taken into account. The IBIS lightcurves of 43 INTEGRAL bursts are given in the Appendix in the $25-50 \mathrm{keV}$ and $50-300 \mathrm{keV}$ energy ranges. In general, the faint bursts have smooth, long-duration profiles with only one or two weak pulses. 
Table 3. IBIS and SPI spectral properties of GRBs for which Band and quasithermal (PL + BB) models are fit to spectra. Parameters quoted are low energy power-law index, $\alpha$, high energy power-law index, $\beta$, and break energy, $E_{0}$, for Band model fits and temperature, $k T$ and power-law index, $\Gamma$ for quasithermal model fits.

\begin{tabular}{|c|c|c|c|c|c|c|c|c|}
\hline \multirow[b]{2}{*}{ GRB } & & \multicolumn{4}{|c|}{$\overline{\text { Band model }}$} & \multicolumn{3}{|c|}{ Quasithermal model } \\
\hline & & $\alpha$ & $\beta$ & $E_{0}(\mathrm{keV})$ & $\chi^{2} /$ d.o.f. & $k T$ & $\Gamma$ & $\chi^{2} /$ d.o.f. \\
\hline$\overline{\text { GRB } 030131}$ & $I B I S$ & $-1.40 \pm 0.20$ & $-3.0 \pm 1.0$ & $70 \pm 20$ & $22.5 / 16$ & - & - & - \\
\hline \multirow[t]{2}{*}{ GRB 040422} & IBIS & $-1.26 \pm 0.08$ & $-3^{\dagger}$ & $55 \pm 31$ & $42.0 / 35$ & $14 \pm 2$ & $-2.49 \pm 0.48$ & $38.4 / 34$ \\
\hline & $S P I$ & & $2.17 \pm 0.28(\mathrm{PL})$ & & $7.5 / 3$ & - & - & - \\
\hline \multirow[t]{2}{*}{ GRB 041218} & $I B I S$ & $-1.15 \pm 0.20$ & $-2.08 \pm 0.16$ & $80 \pm 40$ & $64.4 / 35$ & $14 \pm 2$ & $-1.76 \pm 0.08$ & $64.1 / 35$ \\
\hline & SPI & $-0.97 \pm 0.47^{1}$ & $-3^{\dagger}$ & $116 \pm 152$ & $20.0 / 35$ & $26 \pm 9$ & $-1.82 \pm 0.38$ & $19.4 / 34$ \\
\hline \multirow[t]{2}{*}{ GRB 041219a } & IBIS & $-1.67^{\dagger}$ & $-1.92 \pm 0.02$ & 114 & $184.0 / 157$ & $30 \pm 12$ & $-2.03 \pm 0.07$ & $109.6 / 87$ \\
\hline & $S P I$ & $-1.48 \pm 0.08$ & $-1.92 \pm 0.13$ & $365.9 \pm 192$ & $51.9 / 30$ & $29 \pm 3$ & $-1.77 \pm 0.03$ & $17.6 / 18$ \\
\hline GRB 050502a & $I B I S$ & $-0.89 \pm 0.25$ & $-3^{\dagger}$ & $83 \pm 44$ & $34.7 / 38$ & $17 \pm 2$ & $-1.64 \pm 0.11$ & $33.2 / 41$ \\
\hline \multirow[t]{2}{*}{ GRB 050525a } & IBIS & $-0.94 \pm 0.45$ & $-3^{\dagger}$ & $65 \pm 62$ & $59.2 / 31$ & $18 \pm 4$ & $-2.05 \pm 0.58$ & $60.1 / 31$ \\
\hline & SPI & $-1.37 \pm 0.23$ & $-3^{\dagger}$ & $127 \pm 45$ & $46.9 / 51$ & $26 \pm 4$ & $-2.24 \pm 0.15$ & $46.0 / 50$ \\
\hline \multirow[t]{2}{*}{ GRB 050918} & $I B I S$ & $-1.42 \pm 0.51$ & $-3^{\dagger}$ & $117 \pm 1484$ & $26.4 / 29$ & $21 \pm 5$ & $-2.44 \pm 1.22$ & $23.8 / 28$ \\
\hline & $S P I$ & $-1.28 \pm 2.58$ & $-3^{\dagger}$ & 69 & $4.4 / 7$ & $15 \pm 11$ & $-2.24 \pm 3.22$ & $3.8 / 6$ \\
\hline GRB $060428 \mathrm{c}$ & $I B I S / S P I$ & $-0.71 \pm 0.26$ & -2.0 & $54 \pm 14$ & $32.3 / 33$ & - & 一 & - \\
\hline \multirow[t]{2}{*}{ GRB 060901} & $I B I S$ & $-1.47 \pm 0.20$ & $-3^{\dagger}$ & $998 \pm 9002$ & $40.2 / 30$ & $36 \pm 11$ & $-1.91 \pm 0.39$ & $34.8 / 29$ \\
\hline & $S P I$ & $-0.50 \pm 0.61$ & $-1.89 \pm 0.42$ & $85 \pm 151$ & $9.0 / 14$ & $28 \pm 11$ & $-1.54 \pm 0.15$ & $14.0 / 14$ \\
\hline \multirow[t]{2}{*}{ GRB 061025} & IBIS & $-0.85 \pm 0.43$ & $-3^{\dagger}$ & $94 \pm 134$ & $38.3 / 29$ & $15 \pm 6$ & $-1.38 \pm 0.30$ & $35.6 / 28$ \\
\hline & $S P I$ & & $1.57 \pm 0.25(\mathrm{PL})$ & & $10.4 / 6$ & $17 \pm 6$ & $-0.75 \pm 2.65$ & $7.2 / 5$ \\
\hline \multirow[t]{2}{*}{ GRB 061122} & $I B I S$ & $-0.97 \pm 0.40$ & $-2.00 \pm 0.12$ & $56 \pm 52$ & $47.4 / 30$ & $12 \pm 3$ & $-1.72 \pm 0.12$ & $48.6 / 30$ \\
\hline & $S P I$ & $-0.98 \pm 0.12$ & $-2.72 \pm 0.85$ & $166 \pm 39$ & $66.6 / 56$ & $36 \pm 3$ & $-1.81 \pm 0.08$ & $67.8 / 56$ \\
\hline
\end{tabular}

$\dagger$ Parameter frozen.

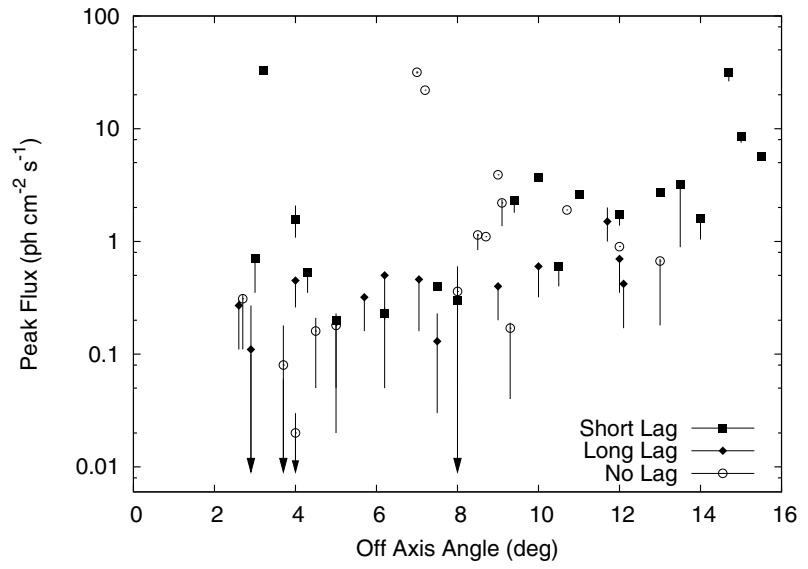

Fig. 4. The off-axis angle distribution of the GRBs in the IBIS FoV as a function of peak flux $(20-200 \mathrm{keV})$. The FCFoV is $9^{\circ} \times 9^{\circ}$ and the boundary of the $\mathrm{PCFoV}$ is between $4.5^{\circ}$ and $6.2^{\circ}$, depending on the azimuthal angle of the GRB position. The long-lag GRBs are marked with filled diamonds. There are 11 GRBs within the FCFoV and 4 have long lags. IBIS is less sensitive to $\gamma$-ray sources outside the FCFoV as reflected by the decrease in the number of faint GRBs.

\subsection{Spectral properties of INTEGRAL GRBS}

The spectral information given in Table 2 is obtained from the IBIS detector, with the exception of GRB 041219a, GRB 050525a, GRB 060901 and GRB 061122 for which the peak flux and fluence values are measured with SPI in the 20-200 keV energy range due to telemetry gaps in the IBIS data (McBreen et al. 2006; McGlynn et al. 2008b). Limited spectral information is available for GRB 021125 due to significant telemetry loss while the satellite was in calibration mode (Malaguti et al. 2003). Data for GRB 060930 and GRB 070311 are currently not publicly available and values shown are taken

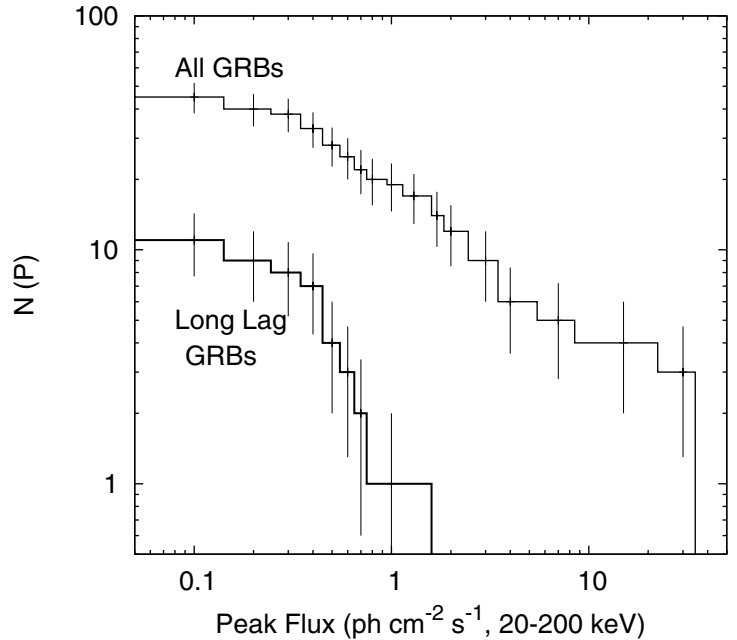

Fig. 5. The cumulative $\log N-\log P$ distribution of the 47 GRBs detected by IBIS, with peak flux $P$ measured between $20-200 \mathrm{keV}$. The distribution is biased by the lower sensitivity of IBIS at large off-axis angles (Fig. 4). The small subset of 11 long-lag GRBs is shown separately.

from Gotz et al. (2006) and Mereghetti et al. (2007), respectively. Peak flux values are integrated over the brightest $1 \mathrm{~s}$ time interval in the 20-200 keV energy band and fluence values are measured over the same energy range. Photon indices are given for a power-law fit to the spectra in the $20-200 \mathrm{keV}$ energy range. Spectral parameters obtained from IBIS and SPI spectra for which the Band model and quasithermal model are fit are listed in Table 3. The values obtained from a combined IBIS and SPI spectral fit to GRB $060428 \mathrm{c}$ over the $20-400 \mathrm{keV}$ energy range are taken from Grebenev \& Chelovekov (2007). GRB 030131 and GRB 050502a were detected during a satellite 


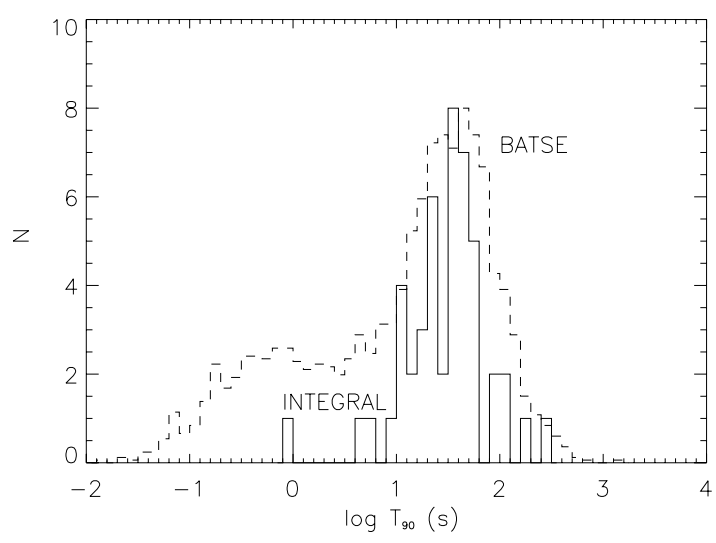

Fig. 6. $T_{90}$ distribution of INTEGRAL GRBs (solid line) in comparison to that of BATSE (dashed line). The BATSE distribution is normalised to the peak of the INTEGRAL distribution for clarity. The BATSE data for 2041 GRBs is taken from the Current Catalog at http://www . batse.msfc.nasa.gov/batse/grb/catalog/current.

slew so SPI spectral analysis was not possible for these bursts. A sample of INTEGRAL $v F_{v}$ spectra is shown in Fig. 7.

The distribution of photon indices is shown in Fig. 8 for INTEGRAL and Swift GRBs for which a power-law model was fit to the spectral data in the $20-200 \mathrm{keV}$ and $15-150 \mathrm{keV}$ energy ranges, respectively. In comparison to Swift, INTEGRAL detects proportionally more soft GRBs with steeper power-law photon indices.

Figure 9 compares the peak flux (20-200 keV) distribution of the GRBs observed by IBIS (solid line) to the peak flux (15-150 keV) distribution of the GRBs detected by the BAT instrument on Swift. IBIS detects proportionally more weak GRBs than Swift because of its better sensitivity within a FoV that is smaller by a factor of $\sim 12$.

\subsection{Spectral lags of INTEGRAL GRBs}

The spectral lag results are presented in Table 4. A positive spectral lag follows the usual convention of high energy emission preceding low energy emission. The time intervals which were correlated to determine the spectral lags are marked with vertical lines on the lightcurves in the Appendix. Within these intervals, only those counts above $10 \%$ of the peak count rate were correlated to give the lag values in Table 4 . The number distribution of spectral lags measured over the full burst duration is given in Fig. 10 for the 28 long-duration GRBs with a measured lag between Channel $1(25-50 \mathrm{keV})$ and Channels 2 and $3(50-300 \mathrm{keV})$. No statistically significant negative spectral lags are found. Negative lags, which violate the typical hardsoft evolution of GRBs, have been observed in a small minority of cases (e.g. Chen et al. 2005) and may be more prevalent in short bursts (Gupta et al. 2002; Yi et al. 2006). A long tail extending to $\sim 5 \mathrm{~s}$ is observed in the lag distribution in Fig. 10 and a clear separation between short and long lag is drawn at $\tau_{2+3,1} \sim 0.75 \mathrm{~s}$. Thus, long-lag bursts have $\tau_{2+3,1}>0.75 \mathrm{~s}$ and those with $\tau_{2+3,1}<0.75 \mathrm{~s}$ are referred to as short-lag GRBs. The 11 long-lag GRBs are identified in Table 4, with 4 in the FCFoV, a further 4 in the PCFoV to the $50 \%$ coding level and the remaining 3 GRBs at larger off-axis angles (Fig. 4).

The spectral lag distribution of INTEGRAL GRBs as a function of peak flux is shown in Fig. 11. The BeppoSAX SN burst GRB 980425 (Galama et al. 1998) is also shown in Fig. 11. The $\mathrm{SN}$ burst XRF 060218, with a peak flux of $0.6 \mathrm{ph} \mathrm{cm}^{-2} \mathrm{~s}^{-1}$, is
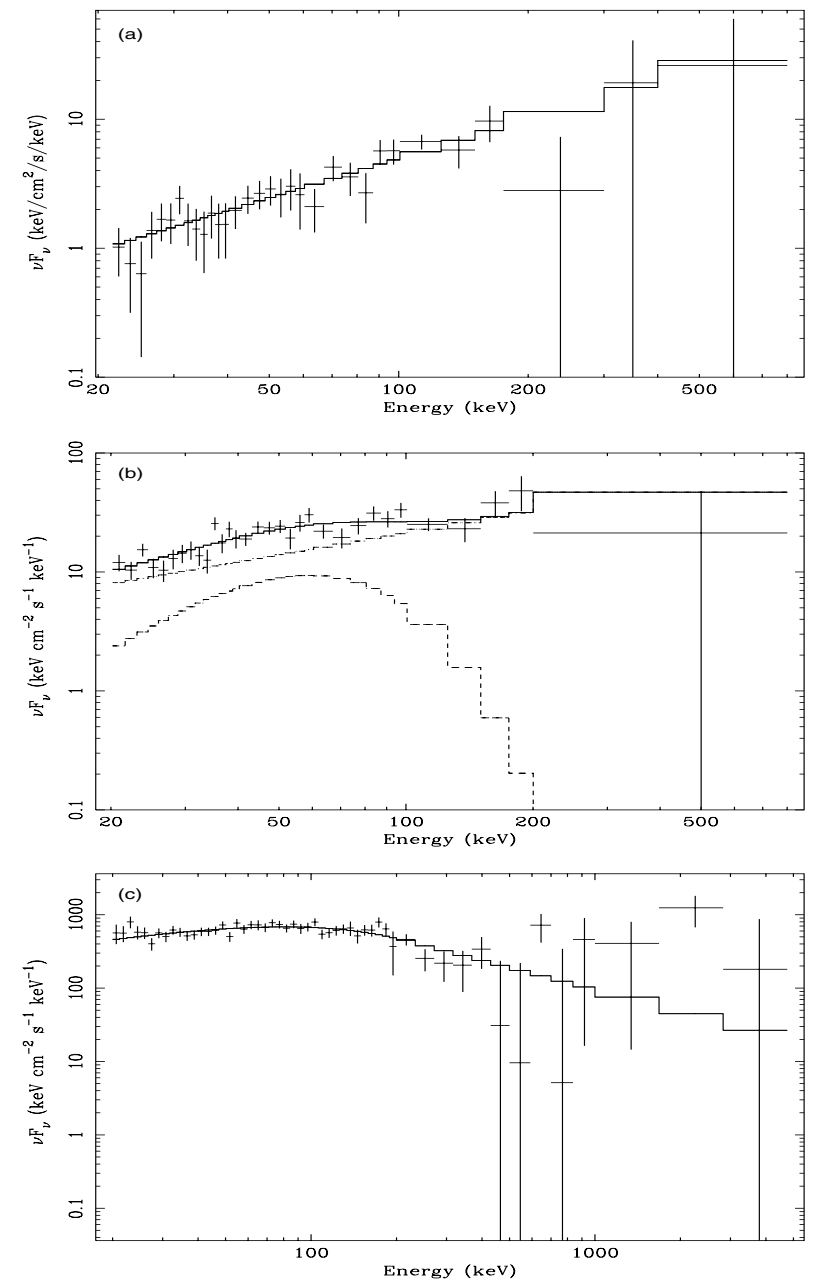

Fig. 7. a) IBIS $v F_{v}$ power-law spectrum of GRB 060114 in the 20-800 keV energy range. b) IBIS $v F_{v}$ spectrum of GRB 061025, fit by the quasithermal (blackbody + power-law) model in the 20-800 keV energy range. c) SPI $v F_{v}$ spectrum of GRB 050525a, from $20 \mathrm{keV}-8 \mathrm{MeV}$, fit with the Band model.

not included in the figure because it has an extremely long lag of $61 \pm 26$ s (Liang et al. 2006). The other low luminosity burst shown in Fig. 11 is GRB060505, which has no associated SN (Fynbo et al. 2006; McBreen et al. 2008). The figure shows that both bright and faint GRBs have short spectral lags, but there is an obvious absence of bright long-lag GRBs. Therefore GRBs with long spectral lags tend to be weak bursts with low peak flux. This trend is in good agreement with that observed using BATSE GRBs (Norris 2002), where the proportion of long-lag GRBs is negligible among bright BATSE bursts and increases to around $50 \%$ at the trigger threshold. Using 1429 BATSE GRBs, Norris (2002) identified three GRB groups that consist of bright shortlag bursts, weak short-lag bursts and weak long-lag bursts. These groups are also clearly identifiable for the INTEGRAL sample in Fig. 11.

The isotropic peak luminosity as a function of spectral lag is shown in Fig. 12 and includes the 3 INTEGRAL GRBs with known redshift for which a lag was measured. The lowluminosity bursts GRB 980425, XRF 060218 and GRB 060505 are also plotted in Fig. 12. The dashed line is the anti-correlation between lag and luminosity proposed by Norris et al. (2000). The bright INTEGRAL bursts GRB 050502a and GRB 050525a have peak luminosities of $1.8 \times 10^{52} \mathrm{erg} \mathrm{s}^{-1}$ and $1.8 \times 10^{51} \mathrm{erg} \mathrm{s}^{-1}$ 


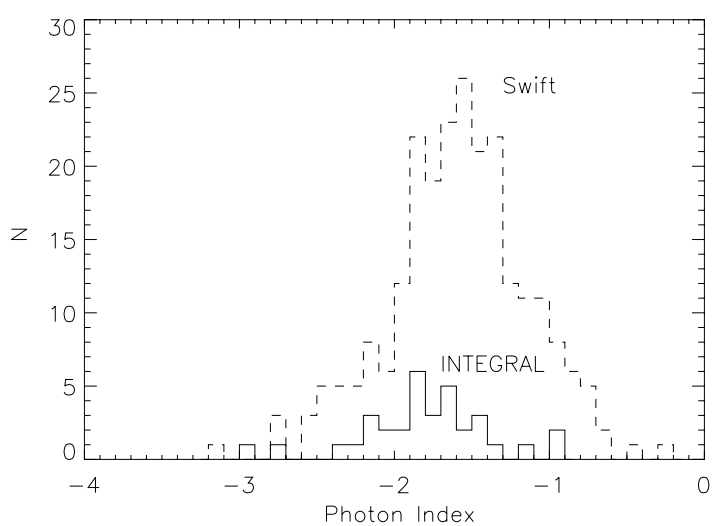

Fig. 8. Power-law photon index distribution for INTEGRAL GRBs detected between October 2002 and July 2007 (solid line) and Swift GRBs detected between November 2004 and July 2007 (dashed line), in the 20-200 keV and 15-150 keV energy bands, respectively. The Swift data for 238 GRBs is taken from http://swift.gsfc.nasa.gov/ docs/swift/archive/grb_table.html.

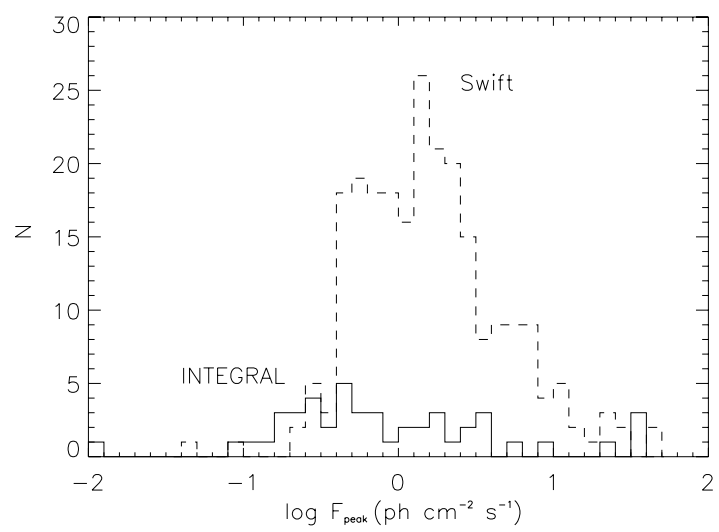

Fig. 9. Peak flux distribution for GRBs detected by INTEGRAL (20-200 keV, solid line) and Swift (15-150 keV, dashed line). The Swift data for 237 GRBs is taken from http://swift.gsfc.nasa.gov/ docs/swift/archive/grb_table.html.

and spectral lags of $0.11_{-0.06}^{+0.07} \mathrm{~s}$ and $0.130_{-0.002}^{+0.003} \mathrm{~s}$, respectively and follow the trend of the relation. At $z=0.106$, GRB 031203 has a peak luminosity of $8.4 \times 10^{48} \mathrm{erg} \mathrm{s}^{-1}$ and spectral lag of $0.17_{-0.04}^{+0.03} \mathrm{~s}$, causing it to fall significantly below the correlation (Sazonov et al. 2004; Gehrels et al. 2006). The HETE burst GRB 030528 has a long lag similar to that of GRB 980425 but a relatively high luminosity and is consistent with the lag-luminosity relation (Gehrels et al. 2006). A long spectral lag is therefore not an exclusive determinant for a lowluminosity GRB.

For the SN burst GRB 031203, spectral lags of $0.24 \pm$ $0.12 \mathrm{~s}$ between $20-50 \mathrm{keV}$ and $100-200 \mathrm{keV}$ (Sazonov et al. 2004 ) and $0.30 \pm 0.20 \mathrm{~s}$ between $15-50 \mathrm{keV}$ and $50-150 \mathrm{keV}$ (Shrader 2006) have been reported and are consistent with the value $\tau_{3,1}=0.17_{-0.04}^{+0.03} \mathrm{~s}$ in Table 4 and Fig. 11. A spectral lag for GRB 040403 of $0.6 \pm 0.1 \mathrm{~s}$ between $15-40 \mathrm{keV}$ and 40-200 keV has previously been reported (Mereghetti et al. 2005), consistent with the value of $\tau_{2+3,1}=0.95_{-0.15}^{+0.25} \mathrm{~s}$ in Table 4, taking into account the different energy ranges. The spectral lag of GRB 050525a was found to be $\tau_{3,1}=0.124 \pm$ $0.006 \mathrm{~s}$ (Norris et al. 2005), in agreement with the value of $\tau_{3,1}=0.130_{-0.002}^{+0.003} \mathrm{~s}$ obtained in Table 4 . The spectral lag for the short burst, GRB 070707 is $5 \pm 5 \mathrm{~ms}\left(\tau_{2+3,1}\right)$, consistent with the negligible lag values expected for short GRBs
(Norris \& Bonnell 2006; Zhang et al. 2006a). For most of the GRBs with distinct separate pulses, the spectral lag does not evolve significantly during the burst, i.e. GRB 030320, GRB 040106, GRB 040422, GRB 040812, GRB 050522 and GRB 050525a. Some evolution is evident in GRB 050918 for which the lag varies from $0.50_{-0.04}^{+0.05} \mathrm{~s}$ for the first pulse of the burst, to a slightly negative lag of $-0.14 \pm 0.01 \mathrm{~s}$ for the second pulse. For GRB 041219a, the lag of the precursor is relatively long and is much shorter for the main emission pulses, as discussed in McBreen et al. (2006). Spectral lag evolution is often seen in multi-peaked GRBs (e.g. Hakkila \& Giblin 2004; Chen et al. 2005; Ryde et al. 2005; Hakkila et al. 2008), and poses obvious difficulties for the use of the lag as a luminosity indicator.

Spectral lags are measured between wavelet-smoothed lightcurves for a number of GRBs with $F_{\text {peak }}(20-200 \mathrm{keV}) \lesssim$ $0.6 \mathrm{ph} \mathrm{cm}^{-2} \mathrm{~s}^{-1}$ and are denoted by $\dagger$ in Table 4 . Table 5 gives the lags determined with and without denoising for these GRBs. The denoising method allows the lag to be measured in some cases for very weak GRBs where it cannot be determined using the raw data and in most cases the lag is better constrained with smaller errors.

Spectral lag measurements were not possible for 16 GRBs for the following reasons:

1. Significant telemetry gaps occur in the IBIS data for GRB 021125 (Fig. A.1a)， GRB 030131， GRB 030227 (Fig. A.1c) and GRB 061122 (Fig. A.1d).

2. GRB 041015 (Fig. A.1f), GRB 050129 (Fig. A.1i), GRB 050922a (Fig. A.1g), GRB 060114 (Fig. A.1j), GRB 060204a (Fig. A.11) and GRB 060912b (Fig. A.1b) are too weak, even when denoised lightcurves are used for the correlation.

3. GRB 050223 (Fig. A.1j), GRB 050714a (Fig. A.1e) and GRB 051105 b (Fig. A.1h) have significant temporal variations which results in incorrect pulses being correlated and so significant CCF peaks were not found for these bursts.

4. IBIS data are currently unavailable to this team for GRB 060428 c, GRB 060930 and GRB 070311.

There are special circumstances for some GRBs and these include:

1. Spectral lags for GRB 021219 (Fig. A.1b) and GRB 050502a (Fig. A.1k) are measured over the region of the burst before telemetry saturation occurred.

2. For GRB 030529 only the first peak is correlated because telemetry gaps occur in the later data (Fig. A.1f).

3. GRB 040903 (Fig. A.1e) and GRB 050522 (Fig. A.1b) are not detected above $50 \mathrm{keV}$ so the lag is determined between 15-25 keV and 25-50 keV (Channel 2).

4. GRB 041219a (Fig. A.1h) (Fenimore et al. 2004) and GRB 050525a (Fig. A.1c) were simultaneously observed by INTEGRAL and Swift. There are telemetry gaps in the IBIS data for both bursts and SPI data types do not permit energy-resolved lightcurves to be extracted. The spectral lags are therefore determined using data from the BAT instrument on Swift in the same energy ranges.

\section{Discussion}

\subsection{INTEGRAL GRBs of particular note}

GRB 031203 is the third nearest GRB at $z=0.1055$ (Prochaska et al. 2004) and is notable for its unambiguous association with 
Table 4. Spectral lag measurements for INTEGRAL GRBs. The columns refer to (from left to right): GRB; supergalactic longitude (SGL) and latitude (SGB); burst interval used in spectral lag determination relative to trigger time and as marked on time profiles in the Appendix; spectral lags measured between Channel $2(50-100 \mathrm{keV})$ and Channel $1(25-50 \mathrm{keV}), \tau_{2,1}$, Channels 2 and 3 combined (50-300 keV) and Channel 1 $(25-50 \mathrm{keV}), \tau_{2+3,1}$ and Channel $3(100-300 \mathrm{keV})$ and Channel $1(25-50 \mathrm{keV}), \tau_{3,1}$.

\begin{tabular}{|c|c|c|c|c|c|c|}
\hline \multirow[t]{2}{*}{$\overline{\overline{\text { GRB }}}$} & \multirow[t]{2}{*}{ 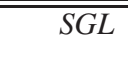 } & \multirow[t]{2}{*}{ 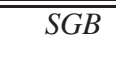 } & \multirow[t]{2}{*}{ Lag interval } & \multicolumn{3}{|c|}{ "Spectral lag } \\
\hline & & & & $\tau_{2,1}(\mathrm{~s})$ & $\tau_{2+3,1}(\mathrm{~s})$ & $\tau_{3,1}(\mathrm{~s})$ \\
\hline$\overline{\text { GRB } 021219}$ & 29.91 & 73.72 & $29.5-33 \mathrm{~s}$ & $0.33 \pm 0.02$ & $0.39 \pm 0.01$ & $0.63 \pm 0.02$ \\
\hline \multirow[t]{3}{*}{ GRB 030320} & -174.44 & 46.17 & $-5-50 s$ & $0.08 \pm 0.03$ & $0.15 \pm 0.03$ & $0.28 \pm 0.03$ \\
\hline & & & $5-20 \mathrm{~s}$ & $0.33 \pm 0.03$ & $0.33 \pm 0.03$ & $0.28 \pm 0.03$ \\
\hline & & & $37-49 s$ & $-0.05 \pm 0.03$ & $0.05 \pm 0.03$ & $0.28_{-0.03}^{+0.05}$ \\
\hline GRB 030501 & -137.97 & 80.21 & $0-30 \mathrm{~s}$ & $-0.30 \pm 0.10$ & $-0.25 \pm 0.10$ & - \\
\hline GRB $030529^{\dagger}$ & 178.44 & -38.74 & $-5-16 s$ & $0.30 \pm 0.30$ & $0.40_{-0.25}^{+0.20}$ & - \\
\hline GRB 031203 & 178.37 & -61.73 & $-5-20 \mathrm{~s}$ & $-0.05_{-0.05}^{+0.04}$ & $0.04 \pm 0.03$ & $0.17_{-0.04}^{+0.03}$ \\
\hline \multirow[t]{3}{*}{ GRB 040106} & 161.11 & -22.05 & $-2-53 \mathrm{~s}$ & $0.00_{-0.05}^{+0.0 .05}$ & $0.05 \pm 0.05$ & -0.04 \\
\hline & & & $-2-10 \mathrm{~s}$ & $0.15_{-0.10}^{-0.05}$ & $0.05_{-0.05}^{+0.10}$ & - \\
\hline & & & $37-53 \mathrm{~s}$ & $-0.35 \pm 0.10$ & $-0.30 \pm 0.05$ & - \\
\hline GRB $040223^{1 \dagger}$ & 179.35 & 24.44 & $-15-15 s$ & $1.05_{-0.35}^{+0.30}$ & $1.80_{-0.25}^{+0.35}$ & - \\
\hline GRB $040323^{1}$ & 170.14 & -3.73 & $0-20 \mathrm{~s}$ & $0.80_{-0.10}^{+0.05}$ & $1.15_{-0.05}^{+0.15}$ & $1.80_{-0.20}^{+0.10}$ \\
\hline GRB $040403^{1 \dagger}$ & 30.70 & -5.66 & $-10-10 \mathrm{~s}$ & $0.60 \pm 0.20$ & $0.95_{-0.15}^{+0.05}$ & - \\
\hline \multirow[t]{3}{*}{ GRB 040422} & -167.01 & 75.91 & $0-10 \mathrm{~s}$ & $0.01 \pm 0.01$ & $0.01 \pm 0.01$ & - \\
\hline & & & $2.5-4 \mathrm{~s}$ & $-0.01 \pm 0.01$ & $-0.01 \pm 0.01$ & - \\
\hline & & & $4-8 \mathrm{~s}$ & $0.04 \pm 0.01$ & $0.02_{-0.02}^{+0.02}$ & - \\
\hline GRB $040624^{2 \dagger}$ & 120.23 & 0.26 & $-5-50 \mathrm{~s}$ & - & $2.75 \pm 0.20$ & - \\
\hline GRB $040730^{3 \dagger}$ & -177.00 & 8.47 & $15-40 \mathrm{~s}$ & $2.80 \pm 0.50$ & $3.05_{-0.45}^{+0.40}$ & $3.30_{-0.35}^{+0.45}$ \\
\hline \multirow[t]{3}{*}{ GRB 040812} & 179.07 & 20.75 & $4-12 \mathrm{~s}$ & $-0.05_{-0.10}^{+0.05}$ & $-0.15 \pm 0.10$ & $-^{-0.35}$ \\
\hline & & & $4-7.7 \mathrm{~s}$ & $0.00 \pm 0.05$ & $0.00 \pm_{-0.15}^{+0.10}$ & - \\
\hline & & & $7.7-12 \mathrm{~s}$ & $-0.20_{-0.10}^{+0.15}$ & $-0.30_{-0.10}^{+0.15}$ & - \\
\hline GRB $040827^{2 \dagger}$ & 144.61 & 27.49 & $5-30 \mathrm{~s}$ & $3.15_{-0.30}^{+0.20}$ & $4.85_{-0.30}^{+0.15}$ & - \\
\hline GRB $040903^{\dagger}$ & -170.84 & 47.15 & $0-20 \mathrm{~s}$ & & $\tau_{1,15-25}=0.10_{-0.15}^{+0.25}$ & \\
\hline \multirow[t]{4}{*}{ GRB 041218} & 7.76 & 11.40 & $20-63 \mathrm{~s}$ & $0.05_{-0.01}^{+0.02}$ & $0.04_{-0.03}^{+0.01}$ & $-0.03_{-0.02}^{+0.04}$ \\
\hline & & & $20-29 \mathrm{~s}$ & $0.24_{-0.04}^{+0.01}$ & $0.15_{-0.06}^{+0.05}$ & $-^{-0.02}$ \\
\hline & & & $31-40 \mathrm{~s}$ & $-0.01_{-0.03}^{-0.02}$ & $-0.02 \pm 0.02$ & $-0.01_{-0.02}^{+0.04}$ \\
\hline & & & $50-63 \mathrm{~s}$ & $-0.02 \pm 0.03$ & $-0.06_{-0.02}^{+0.04}$ & $-^{-0.02}$ \\
\hline \multirow[t]{4}{*}{ GRB 041219a } & -1.86 & 17.42 & $261-414 \mathrm{~s}$ & $0.12 \pm 0.02$ & $0.15 \pm 0.02$ & $0.21 \pm 0.02$ \\
\hline & & & $-1-7 \mathrm{~s}$ & $0.50_{-0.08}^{+0.50}$ & $0.65_{-0.06}^{+0.05}$ & $1.00_{-0.09}^{+0.10}$ \\
\hline & & & $261-327 \mathrm{~s}$ & $0.06 \pm 0.02$ & $0.08 \pm 0.02$ & $0.14 \pm 0.02$ \\
\hline & & & $356-414 \mathrm{~s}$ & $0.10 \pm 0.02$ & $0.13 \pm 0.03$ & $0.18 \pm 0.03$ \\
\hline GRB 050502a & 75.87 & 16.88 & $-16.5--3.5 \mathrm{~s}$ & $-0.12_{-0.08}^{+0.05}$ & $-0.03 \pm 0.06$ & $0.11_{-0.06}^{+0.07}$ \\
\hline GRB $050504^{3}$ & 77.79 & 15.58 & $-5-60 s$ & $1.35_{-0.20}^{+0.30}$ & $2.40_{-0.25}^{+0.35}$ & $4.35_{-0.70}^{+1.45}$ \\
\hline GRB 050520 & 86.68 & 6.85 & $0-80 \mathrm{~s}$ & $0.07 \pm 0.02$ & $0.07 \pm 0.02$ & - \\
\hline \multirow[t]{3}{*}{ GRB $050522^{\dagger}$} & 93.81 & 12.17 & $0-20 \mathrm{~s}$ & & $\tau_{1,15-25}=0.70_{-025}^{+0.15}$ & \\
\hline & & & $2.5-6 \mathrm{~s}$ & & $\tau_{1,15-25}=0.80_{-0.50}^{+0.75}$ & \\
\hline & & & $7-16 \mathrm{~s}$ & & $\tau_{1,15-25}=0.70_{-0.20}^{+0.15}$ & \\
\hline \multirow[t]{3}{*}{ GRB 050525a } & 51.74 & 78.15 & $0-12 \mathrm{~s}$ & $0.056 \pm 0.001$ & $0.069 \pm 0.001$ & $0.130_{-0.002}^{+0.003}$ \\
\hline & & & $0-4 \mathrm{~s}$ & $0.053 \pm 0.001$ & $0.068 \pm 0.001$ & $0.131_{-0.002}^{+0.002}$ \\
\hline & & & $4-12 \mathrm{~s}$ & $0.066_{-0.002}^{+0.003}$ & $0.068 \pm 0.002$ & $0.096_{-0.005}^{+0.003}$ \\
\hline GRB $050626^{3 \dagger}$ & 178.46 & -17.14 & $50-110 \mathrm{~s}$ & $3.35_{-0.40}^{+0.002}$ & $3.25_{-045}^{+0.35}$ & $\underline{-}^{-0.003}$ \\
\hline \multirow[t]{3}{*}{ GRB 050918} & -174.74 & 45.97 & $0-325 \mathrm{~s}$ & $0.17_{-0.03}^{+0.04}$ & $0.17_{-0.02}^{-0.03}$ & - \\
\hline & & & $0-50 \mathrm{~s}$ & $0.43_{-0.05}^{+0.03}$ & $0.50_{-0.04}^{+0.02}$ & - \\
\hline & & & $220-350 \mathrm{~s}$ & $-0.06 \pm 0.01$ & $-0.14 \pm 0.01$ & - \\
\hline GRB $051211 b^{2}$ & -8.74 & 28.77 & $10-80 \mathrm{~s}$ & $1.95 \pm 0.35$ & $1.70_{-0.35}^{+0.30}$ & $1.55_{-0.35}^{+0.65}$ \\
\hline GRB $060130^{3 \dagger}$ & 163.67 & 16.50 & $-10-45 \mathrm{~s}$ & $4.05_{-0.30}^{+0.15}$ & $3.45_{-0.45}^{+0.35}$ & 一 \\
\hline GRB 060901 & -144.72 & 67.40 & $0-4 \mathrm{~s}$ & $0.2 \pm 0.1$ & $0.2 \pm 0.1$ & $0.2 \pm 0.1$ \\
\hline GRB 061025 & -140.43 & 24.19 & $-10-10 \mathrm{~s}$ & $0.35_{-0.05}^{+0.10}$ & $0.40_{-0.10}^{+0.05}$ & $0.40 \pm 0.05$ \\
\hline GRB $070309^{1 \dagger}$ & -172.41 & 33.13 & $-25-10 \mathrm{~s}$ & - & $\begin{array}{r}-7.10 \\
1.70_{-0.30}^{+0.35}\end{array}$ & - \\
\hline GRB $070615^{\dagger}$ & -59.34 & -30.58 & $0-20 \mathrm{~s}$ & $0.70_{-0.15}^{+0.25}$ & $0.40_{-0.20}^{+0.15}$ & - \\
\hline GRB $070707^{*}$ & -159.26 & 4.64 & $0.4-1.5 \mathrm{~s}$ & $0.025 \pm 0.005$ & $0.005 \pm 0.005$ & $0.020 \pm 0.005$ \\
\hline
\end{tabular}

${ }^{1}$ Long-lag GRB in PCFoV to 50\% coding. ${ }^{2}$ Long-lag GRB in PCFoV outside 50\% coding. ${ }^{3}$ Long-lag GRB in FCFoV. $\dagger$ Lag measured using wavelet-smoothed lightcurves. * Short-duration GRB. 


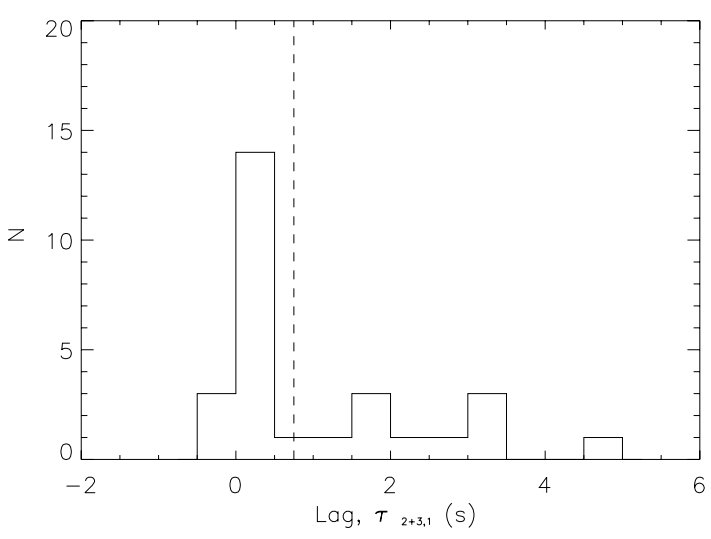

Fig. 10. Spectral lag distribution for the 28 INTEGRAL GRBs for which a lag could be measured between $25-50 \mathrm{keV}$ and $50-300 \mathrm{keV}\left(\tau_{2+3,1}\right)$. The distribution is separated by the dashed line into short-lag and longlag GRBs at $\tau_{2+3,1}=0.75 \mathrm{~s}$.

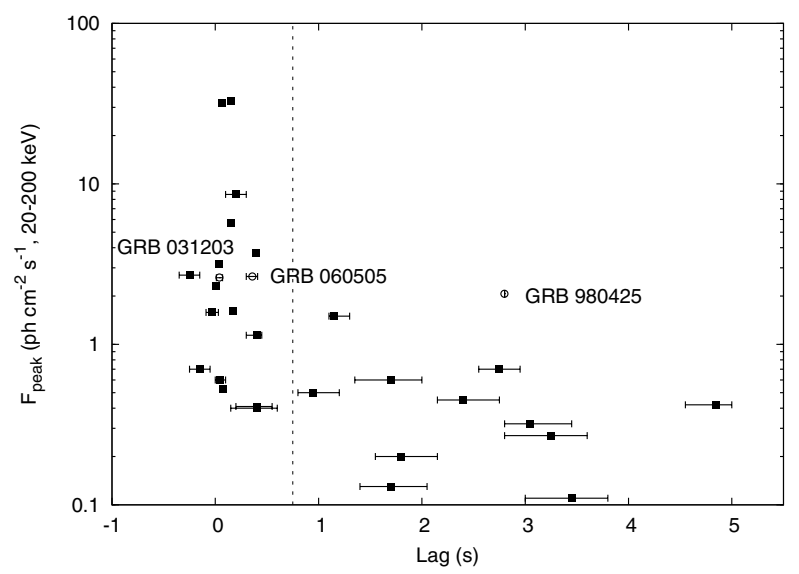

Fig. 11. Spectral lag distribution of INTEGRAL GRBs as a function of peak flux (20-200 keV). The SN bursts GRB 980425 and GRB 031203 are identified and represented by open circles, as is GRB 060505 which does not have an associated SN. XRF060218 has a peak flux of $0.6 \mathrm{ph} \mathrm{cm}^{-2} \mathrm{~s}^{-1}$ and is not included in the figure because of its very long lag of $61 \pm 26 \mathrm{~s}$. GRBs with the longest lags tend to have low peak fluxes, whereas GRBs with short lags have both low and high peak fluxes. The dashed line indicates the separation between long and shortlag GRBs at $\tau_{2+3,1}=0.75 \mathrm{~s}$.

the supernova SN 20031w (Malesani et al. 2004). It is a confirmed low-luminosity GRB with an isotropic energy of $\sim 4 \times$ $10^{49}$ erg (20-200 keV) (Sazonov et al. 2004) and a lag of $0.17_{-0.03}^{+0.04} \mathrm{~s}$ between $25-50$ and $50-300 \mathrm{keV}$. The IBAS localisation of GRB 031203 enabled XMM-Newton to begin followup observations $6 \mathrm{~h}$ later (Santos-Lleo et al. 2003). The X-ray observations showed concentric ring-like structures centred on the GRB location, making this the first detection of a GRB $\mathrm{X}$-ray halo, caused by X-ray scattering from dust columns in our galaxy along the line of sight to the GRB (Vaughan et al. 2004; Tiengo \& Mereghetti 2006). Dust scattering X-ray halos have recently been observed for two Swift GRBs (Vianello et al. 2007). The X-ray observations of GRB 031203 inferred a very high soft $\mathrm{X}$-ray flux for this burst, implying that this GRB may have been an X-ray Flash (XRF) (Watson et al. 2004, 2006; Sazonov et al. 2006).

GRB 041219a is the brightest burst localised by INTEGRAL (McBreen et al. 2006). The peak flux of $43 \mathrm{ph} \mathrm{cm}^{-2} \mathrm{~s}^{-1}$ ( $20 \mathrm{keV}-8 \mathrm{MeV}, 1 \mathrm{~s}$ integration) is greater than that for $\sim 98 \%$ of all bursts and the total duration of $\sim 520 \mathrm{~s}$ is longer than all

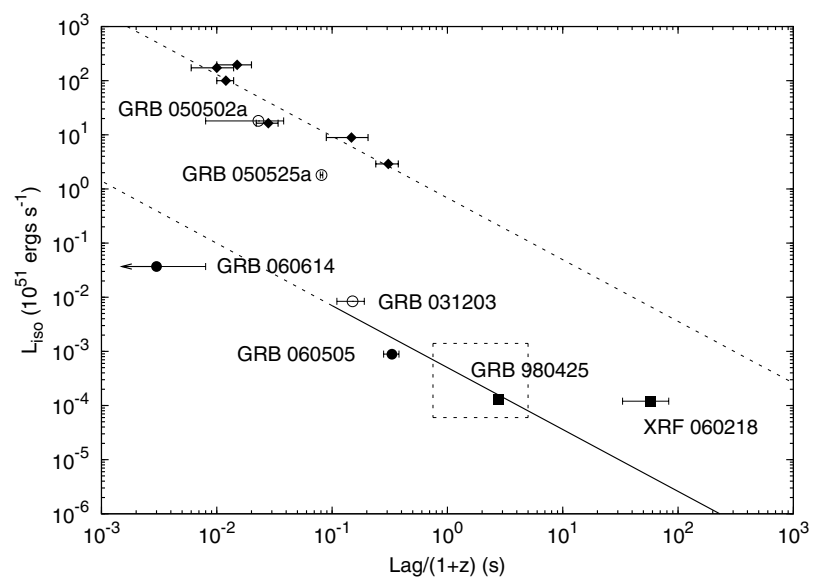

Fig. 12. Isotropic peak luminosity $\left(\times 10^{51} \mathrm{erg} \mathrm{s}^{-1}, 50-300 \mathrm{keV}\right)$ as a function of spectral lag measured between $25-50 \mathrm{keV}$ and 100-300 keV. The two exceptions are GRB 060505 and GRB 060614 for which the lag was measured between $25-50 \mathrm{keV}$ and $50-100 \mathrm{keV}$ and $15-25 \mathrm{keV}$ and $50-100 \mathrm{keV}$, respectively. The dashed line is the anti-correlation obtained for 6 bursts (diamonds) at known redshift (Norris et al. 2000). The 3 INTEGRAL GRBs with a redshift and measured lag are represented by open circles, including the $\mathrm{SN}$ burst GRB 031203. The other low-luminosity SN bursts GRB 980425 and XRF060218 are identified and represented by squares and the two GRBs without SNe by filled circles. The long-lag GRBs lie within the dashed box if they are at the adopted distance of $250 \mathrm{Mpc}$, with peak luminosities ranging from $6 \times 10^{46}$ to $1.4 \times 10^{48} \mathrm{erg} \mathrm{s}^{-1}$ and total luminosities from $10^{48}$ to $10^{50} \mathrm{erg}$. The solid line is the proposed lagluminosity relationship for the low-luminosity GRBs and extrapolates to short GRBs at low values of the lag, e.g. GRB 060614, which lies in the region of the plot occupied by short GRBs. No $k$-correction was applied to the data.

but a small number of bursts. The SPI instrument was used to measure GRB polarisation through multiple scattering events in its $19 \mathrm{Ge}$ detectors (Kalemci et al. 2004), since the scatter angle distribution depends on the polarisation of the incoming photons (Lei et al. 1997). A search for linear polarisation in the most intense pulse (66 s) in GRB 041219a and sub-intervals was performed. Kalemci et al. (2007) and McGlynn et al. (2007) have shown that there is evidence for a high degree of polarisation, but at a low significance level.

\subsection{Afterglows of INTEGRAL GRBs}

X-ray observations were performed for 2 long-lag GRBs with XMM-Newton (Gonzalez-Riestra \& Rodriguez-Pascual 2004; Rodriguez-Pascual \& Gonzalez-Riestra 2004) and 5 long-lag GRBs with Swift (Kennea et al. 2005; Mangano et al. 2005; Parola et al. 2005; Kennea et al. 2006; Vergani et al. 2007). There were also X-ray observations of 8 of the GRBs with no lag measurement. The median X-ray flux for the long-lag GRBs is $\sim 4 \times 10^{-13} \mathrm{erg} \mathrm{cm}^{-2} \mathrm{~s}^{-1}$ between 5 to $10 \mathrm{~h}$ after the burst, with an upper limit in the two cases of non-detection of $\sim 4 \times$ $10^{-14} \mathrm{erg} \mathrm{cm}^{-2} \mathrm{~s}^{-1}$, indicating that the long-lag weak GRBs tend to have a weak X-ray afterglow component. In contrast, the other INTEGRAL GRBs with a measured afterglow have a typical flux of $\sim 9 \times 10^{-13} \mathrm{erg} \mathrm{cm}^{-2} \mathrm{~s}^{-1}$ between 2 and $12 \mathrm{~h}$ after the burst.

Of 300 GRBs localised by Swift up to November 2007, $\sim 60 \%$ have optical or near-IR afterglows and only $\sim 30 \%$ have measured redshifts (Coward et al. 2008), even though deep observations down to $\sim 21-22 \mathrm{mag}$ are carried out for most events within $24 \mathrm{~h}$ of the burst. Dark GRBs without a detectable 
Table 5. Wavelet-denoised spectral lags of weak GRBs. The lags derived from raw data are shown for comparison where available. Spectral lags measured between Channels 2 and 3 combined (50-300 keV) and Channel $1(25-50 \mathrm{keV}), \tau_{2+3,1}$, with the exception of the XRFs for which the lag is measured between $15-25 \mathrm{keV}$ and $25-50 \mathrm{keV}$ (Channel 1), $\tau_{1,15-25}$.

\begin{tabular}{ccc}
\hline \hline GRB & $\tau_{2+3,1}$ (Raw data) (s) & $\tau_{2+3,1}$ (Denoised data) (s) \\
\hline GRB 030529 & - & $0.40_{-0.25}^{+0.20}$ \\
GRB 040223 & $1.70_{-0.25}^{+0.30}$ & $1.80_{-0.25}^{+0.35}$ \\
GRB 040403 & $1.05_{-0.30}^{+0.55}$ & $0.95_{-0.15}^{+0.25}$ \\
GRB 040624 & $3.10_{-0.30}^{+0.90}$ & $2.75 \pm 0.20$ \\
GRB 040730 & $3.35_{-0.55}^{+0.80}$ & $3.05_{-0.45}^{+0.40}$ \\
GRB 040827 & $3.65 \pm 0.30$ & $4.85_{-0.30}^{+0.15}$ \\
XRF 040903 & - & $\tau_{1,15-25}=0.10_{-0.15}^{+0.25}$ \\
XRF 050522 & $\tau_{1,15-25}=1.15_{-0.45}^{+0.25}$ & $\tau_{1,15-25}=0.70_{-0.25}^{+0.15}$ \\
GRB 050626 & $3.25_{-0.65}^{+1.25}$ & $3.25_{-0.45}^{+0.35}$ \\
GRB 060130 & $3.65_{-0.75}^{+1.00}$ & $3.45_{-0.45}^{+0.35}$ \\
GRB 070309 & - & $1.70_{-0.30}^{+0.35}$ \\
GRB 070615 & $0.40_{-0.25}^{+0.15}$ & $0.40_{-0.20}^{+0.15}$ \\
\hline
\end{tabular}

afterglow may therefore make up a significant proportion of the GRB population.

Almost $70 \%$ (31/46) of the GRBs observed by INTEGRAL do not have a detected optical counterpart, including 9 of the 11 long-lag GRBs. The optical observations revealed faint afterglows for GRB 040323 and GRB 040827, and near-IR afterglows for GRB 040223 and GRB 040624 (Filliatre et al. 2006). A non-spectroscopic redshift in the range $0.5<z<1.7$ was obtained for GRB 040827 (de Luca et al. 2005). The IBIS error box of GRB 060114 contains galaxies from the cluster A1651 $(z=0.087)$ and the optical afterglow was fainter than $R=19$ just 1.9 min after the GRB (Guidorzi et al. 2006). Only one radio afterglow has been detected for the long-lag GRBs (Frail 2005), compared to a total of 8/46 for all of the INTEGRAL GRBs.

GRB 040223 and GRB 040624 (Filliatre et al. 2006) provide good examples of GRBs with dark or faint optical afterglows. GRB 040223 was observed close to the galactic plane, so NIR observations were carried out to overcome the high dust obscuration. Observations were undertaken at the NTT of ESO, $17 \mathrm{~h}$ after the GRB and no afterglow was found. GRB 040624 was located far from the galactic plane at high latitude where the optical extinction is negligible. Afterglow observations were carried out $13 \mathrm{~h}$ after the burst using the VLT and TNG. Magnitude limits were obtained in the optical that are fainter than the very faint end of the distribution of the magnitudes of a compilation of 39 promptly observed counterparts. The position of GRB 040624 is less than $5^{\prime}$ from a galaxy in the cluster A1651. A search for a supernova was carried out up to a month after the GRB but none was found to a faint limit of $R>22.6$ (D'Avanzo et al. 2004).

The lack of a detected optical afterglow may be due to a number of factors. Possible explanations include dust obscuration, a low-density environment, an intrinsically faint afterglow in the optical, a rapidly decaying afterglow or the burst occuring at a high redshift (e.g. Jakobsson et al. 2004; Rol et al. 2005). Dust obscuration may be due to a burst environment with a high gas column density (e.g. Lamb 2001; Castro-Tirado et al. 2007) or dust in the host galaxy along the line of sight to the GRB ( $\sim 10 \%$ of dark events, Piro et al. 2002). Low-density GRB environments can also produce a very faint optical afterglow but the association between GRBs and core-collapse SNe does not favour this scenario (Taylor et al. 1998). Some GRBs have intrinsically faint afterglows (Fynbo et al. 2001). GRBs at high redshift can only account for $\sim 10 \%$ of these dark bursts (e.g. Gorosabel et al. 2004; Castro-Tirado et al. 2006). Using early observations of Swift GRBs, Roming et al. (2006) found that $\sim 25 \%$ of the sample were extincted by galactic dust, $\sim 25 \%$ by absorption in the local burst environment and $\sim 30 \%$ were most probably affected by Ly- $\alpha$ absorption at high redshift.

\subsection{INTEGRAL spectra}

Most GRB continuum spectra can be fit by the Band model (Band et al. 1993), an empirical function comprising two smoothly broken power laws. The distributions of the low energy power-law photon index and high energy power-law photon index are distributed around values of $\alpha=-1$ and $\beta=-2.2$, respectively, for time-resolved spectra of 156 GRBs detected by BATSE (Preece et al. 2000). The majority of GRBs have low energy power-law spectral indices in the range $-2<\alpha<0$ (e.g. Preece et al. 2000). Of the 10 INTEGRAL GRBs fit by the Band model (Table 3), all have low energy spectral indices consistent with synchrotron emission, i.e. $-3 / 2<\alpha<-2 / 3$.

A thermal component of the prompt emission spectra has been proposed by several authors (e.g. Ghirlanda et al. 2003; Kaneko et al. 2006; Ryde 2005; Bosnjak et al. 2006; McBreen et al. 2006). In a study of BATSE GRBs with very hard spectra, Ghirlanda et al. (2003) found that the time-resolved spectra were not adequately described by non-thermal emission models and that the early parts of the bursts were well fit by a blackbody component. Ryde (2005) has shown that GRB spectra may be composed of a thermal and a power-law component. McBreen et al. (2006) found that for quasithermal model fits to the precursor and main emission of the INTEGRAL burst GRB 041219a, the blackbody component is more dominant in the precursor of the burst, while the main burst emission is well fit by both the Band and quasithermal models. The blackbody temperature decreases from the precursor of GRB 041219a to the main burst emission as expected from Ryde (2005). The 10 bright GRBs in Table 3 are equally well fit by the Band model and blackbody + power-law model fits to the average prompt emission spectra, with similar $\chi^{2}$ values.

Many of the INTEGRAL GRBs in Table 2 have steep powerlaw spectra, which are significantly outside the usual range of the Band model low-energy spectral index of $-3 / 2<\alpha<-2 / 3$. For these GRBs, the power-law index is similar to the high energy photon index above the break energy, with typical values of $\beta$ between -2 and -2.5 (Preece et al. 2000). If the steep powerlaw indices are assumed to be $\beta$, the break energy, $E_{0}$, must be at or below the sensitivity threshold of IBIS, $\sim 20 \mathrm{keV}$. There is therefore an excess of counts in the X-ray/soft $\gamma$-ray region, implying that they are X-ray rich. The peak energy is given by $E_{\text {peak }}=(2+\alpha) E_{0}$ and has a value of $E_{\text {peak }}=E_{0} / 2$ for a typical value of $\alpha=-1.5$. These GRBs have a low $E_{\text {peak }}$ and will have a low luminosity if they are at low redshift (Amati et al. 2007).

INTEGRAL has detected a number of X-ray rich GRBs. X-ray flashes, X-ray rich GRBs and classical GRBs appear to possess a continuum of spectral properties and it is probable that they have a similar origin (Sakamoto et al. 2005). INTEGRAL $\mathrm{X}$-ray rich bursts tend to be weak and their time profiles consist of long, slow pulses (see Appendix). Examples include GRB 040223 (Fig. A.1i), GRB 040624 (Fig. A.1a), GRB 050626 (Fig. A.1d) and GRB 060130 (Fig. A.1k). 
Table 6. Median properties of the 28 long-duration GRBs with a measured $\tau_{2+3,1}$, categorised into those with short lags $\left(\tau_{2+3,1}<0.75 \mathrm{~s}\right)$ and long lags $\left(\tau_{2+3,1}>0.75 \mathrm{~s}\right.$. $)$

\begin{tabular}{ccc}
\hline \hline & Short-lag GRBs & Long-lag GRBs \\
\hline Fraction of INTEGRAL GRBs & $17 / 28$ & $11 / 28$ \\
Median $\tau_{2+3,1}(\mathrm{~s})$ & 0.07 & 1.70 \\
Median $T_{90}(\mathrm{~s})$ & 27 & 50 \\
Median $F_{\text {peak }}\left(\mathrm{erg} \mathrm{cm}^{-2} \mathrm{~s}^{-1}\right)$ & $2.0 \times 10^{-7}$ & $4.0 \times 10^{-8}$ \\
Median Fluence $\left(\mathrm{erg} \mathrm{cm}^{-2}\right)$ & $2.0 \times 10^{-6}$ & $7.6 \times 10^{-7}$ \\
$\%$ within $\pm 30^{\circ} \mathrm{SGB}$ & $41 \%$ & $91 \%$ \\
\hline
\end{tabular}

\subsection{The rate of INTEGRAL GRBs}

GRBs detected at off-axis angles outside the FCFoV of IBIS have a higher peak flux (Fig. 4) because of the reduction in collecting area. For this reason, we estimate the rate of GRBs both in the $\mathrm{FCFOV}$ and in the PCFoV at greater than $50 \%$ coding. There are 11 GRBs within the FCFoV of $0.025 \mathrm{sr}$ of IBIS in 4 years of observation time, yielding an all-sky rate of $\sim 1400 \mathrm{yr}^{-1}$ above the threshold of $\sim 0.15 \mathrm{ph} \mathrm{cm}^{-2} \mathrm{~s}^{-1}$ in the energy range $20-200 \mathrm{keV}$. The 4 long-lag GRBs in the FCFoV give a rate of $\sim 500 \mathrm{yr}^{-1}$. The $33 \mathrm{GRBs}$ within the PCFoV to the $50 \%$ coding level yield an all-sky rate of $\sim 930 \mathrm{yr}^{-1}$ and $\sim 230 \mathrm{yr}^{-1}$ for the 8 long-lag GRBs above the higher threshold of $\sim 0.25 \mathrm{ph} \mathrm{cm}^{-2} \mathrm{~s}^{-1}$. The long-lag GRBs contribute significantly at faint flux levels and appear to form a separate population in the $\log N-\log P$ distribution (Fig. 5). The rate of GRBs is in good agreement with the values obtained from the more sensitve analysis of BATSE archival data (Kommers et al. 2001; Stern et al. 2001).

\subsection{The population of long-lag GRBs with low luminosity}

The lack of redshift determinations for the weak GRBs detected by IBIS prohibits progress by using individual GRBs. However there are a number of redshift indicators that can be used on weak GRBs. In this case the best redshift indicator is the spectral lag which combines the spectral and temporal properties of the prompt GRB emission. GRBs have a long lag when a typical value of $0.1 \mathrm{~s}$ is redshifted by a large factor or alternatively is an intrinsic property of a low-luminosity GRB such as GRB 980425 and XRF 060218. The rate of $z>5$ GRBs in IBIS has been modelled (Salvaterra et al. 2008; Gorosabel et al. 2004; Guetta \& Piran 2007; Lapi et al. 2008) and is unlikely to be more than 1 or 2 GRBs in 5 years of observations. The long lag is therefore taken to be an intrinsic property of most of the longlag GRBs indicating their low luminosity and we investigate the consequences.

The median properties of long and short lag INTEGRAL GRBs are given in Table 6 for the 28 bursts with a measured value of $\tau_{2+3,1}$. Approximately $40 \%$ of INTEGRAL GRBs with a measured lag belong to the long-lag category. The median peak flux for long-lag GRBs is a factor of $\sim 5$ lower than for GRBs with short lags.

The distribution of the INTEGRAL GRBs in supergalactic coordinates is shown in Fig. 13. All of the INTEGRAL GRBs are divided almost equally between the half of the sky above and below $\pm 30^{\circ}$, in agreement with the exposure map which has $\sim 52 \%$ of the exposure time within $\pm 30^{\circ}$ of the supergalactic plane. However, 10 of the 11 long-lag GRBs are concentrated at supergalactic latitudes between $\pm 30^{\circ}$. The quadrupole moment (Hartmann et al. 1996) has a value of $Q=0.007 \pm$ 0.043 for all INTEGRAL GRBs and $Q=-0.225 \pm 0.090$ for
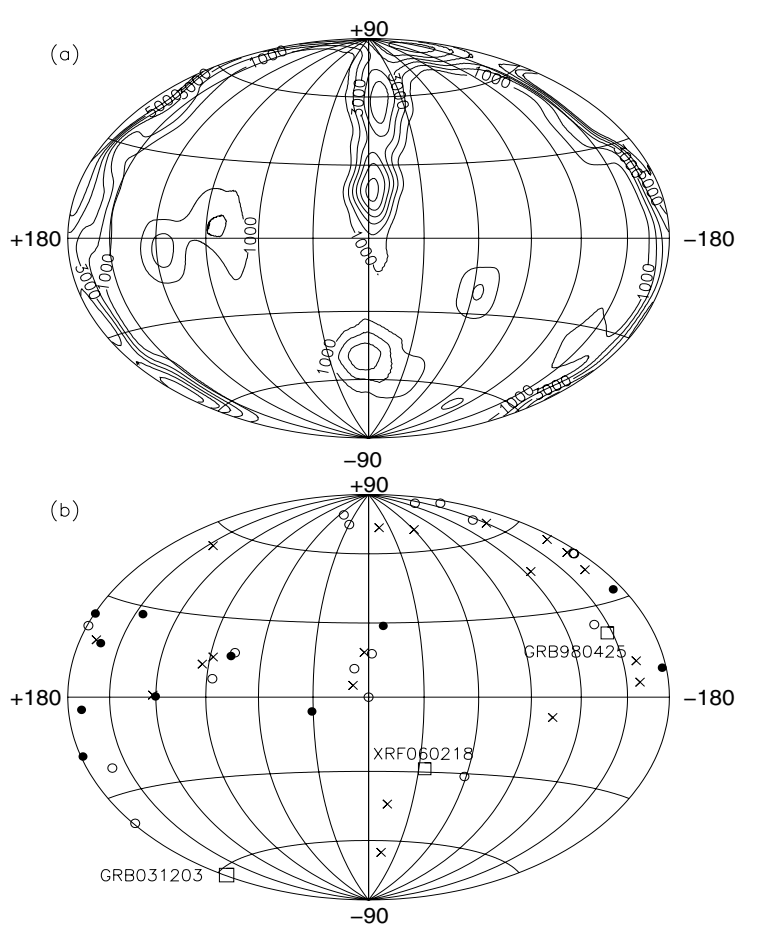

Fig. 13. a) INTEGRAL exposure map in supergalactic co-ordinates up to July 2007 (contours in units of kiloseconds). b) The distribution of INTEGRAL GRBs in supergalactic co-ordinates; the open circles represent short-lag GRBs $\left(\tau_{2+3,1}<0.75 \mathrm{~s}\right)$ and filled-in circles those GRBs with long lags $\left(\tau_{2+3,1}>0.75 \mathrm{~s}\right)$. The 16 GRBs for which a lag could not be determined are denoted by an " $x$ ", as are the XRFs which do not have a measured lag between $25-50 \mathrm{keV}$ and $50-300 \mathrm{keV}$, and the short burst GRB 070707. Ten out of the 11 long-lag GRBs are within $\pm 30^{\circ}$ of the supergalactic plane.

the long-lag GRBs. The quadrupole moment of the 47 bursts is consistent with zero and an isotropic distribution. The nonzero moment of the long-lag bursts indicates an anisotropy in the distribution of these GRBs with respect to the supergalactic plane. The binomial probability that this is a chance occurrence is $7 \times 10^{-3}$. GRB 980425 and XRF 060218 have long lags and lie within $\pm 30^{\circ}$ of the supergalactic plane, while GRB 031203 has a relatively short lag and lies at a high supergalactic latitude. The long-lag GRBs observed with BATSE are also significantly concentrated in the direction $\pm 30^{\circ}$ of the supergalactic plane with a quadrupole moment $Q=-0.097 \pm 0.038$ (Norris 2002). The combined results of more than 14 years of observations with IBIS and BATSE lead us to conclude that long-lag GRBs trace the features of the nearby large-scale structure of the Universe as revealed with superclusters, galaxy surveys (Lahav et al. 2000; Stoughton et al. 2002) and the very high energy cosmic rays (Abraham et al. 2007). This result is a further indication that most long-lag GRBs are nearby and have low luminosity. The local supercluster seems to be appended to a web of filaments and sheets, rather than an isolated pancake structure, with superclusters evident to $\sim 400 \mathrm{Mpc}$. It has been pointed out that weak BATSE GRBs appear to be correlated with galaxies out to distances of $\sim 155 \mathrm{Mpc}$ with the limit determined by galaxy surveys (Chapman et al. 2007).

A nearby population of long-lag, low-luminosity GRBs has previously been proposed based on the detections of GRB 980425, GRB 031203 and XRF 060218 (e.g. Guetta et al. 2004; Daigne \& Mochkovitch 2007). GRB 980425 has an isotropic-equivalent $\gamma$-ray energy release of $\sim 10^{48} \mathrm{erg}$, 
approximately 3 orders of magnitude lower than that of "standard" GRBs, assuming its association with SN 1998bw (Galama et al. 1998) and redshift of $z=0.0085$ (Tinney et al. 1998). XRF 060218, the second closest GRB localised to date, was detected at a redshift of $z=0.033$ (Mirabal et al. 2006). This burst had an extremely long duration $\left(T_{90} \sim 2100 \mathrm{~s}\right)$, a very low isotropic energy of $\sim 6 \times 10^{49} \mathrm{erg}$ and is classified as an XRF (Campana et al. 2006). The distance to the long-lag GRBs can also be constrained by association and comparison with the two low luminosity bursts GRB $980425(\tau \sim 2.8 \mathrm{~s})$ and XRF $060218(\tau \sim 60 \mathrm{~s})$. The distances to GRB 980425 (Galama et al. 1998) and XRF 060218 (Soderberg et al. 2006) are $36 \mathrm{Mpc}$ and $145 \mathrm{Mpc}$, respectively, and these bursts would have been detected in the FCFoV of IBIS to $135 \mathrm{Mpc}$ and $290 \mathrm{Mpc}$. The association of the long-lag GRBs with the known low luminosity GRBs and with the supergalactic plane implies that they are at similar distances. GRB 060505 has no SN to faint limits and has a smaller lag of $\sim 0.4 \mathrm{~s}$ and a distance of $404 \mathrm{Mpc}$ (Fynbo et al. 2006; McBreen et al. 2008).

The region marked with a box on the lag-luminosity plot (Fig. 12) contains the long-lag GRBs if they belong to the low luminosity population at an adopted distance of $250 \mathrm{Mpc}$. The box contains the prototype low luminosity GRB 980425 and is bracketed on one side by GRB 060218 and on the other by GRB $060505(\tau \sim 0.4 \mathrm{~s})$ and GRB $031203(\tau \sim 0.17 \mathrm{~s})$ and at short lag by GRB 060614 which lies in the region occupied by short GRBs (Gehrels et al. 2006). With a long lag of $\sim 2.8 \mathrm{~s}$, GRB 980425 qualitatively follows the lag-luminosity trend but falls significantly below the relation in Fig. 12 (Norris 2002), as do GRB 031203 (Sazonov et al. 2004) and GRB 060218 (Liang et al. 2006). The solid line in Fig. 12 is the proposed lagluminosity relationship for low-luminosity GRBs and is parallel to but shifted from the corresponding fit for the long cosmological GRBs by a factor of $\sim 10^{3}$.

Both GRB 980425 and GRB 031203 violate the Amati correlation (Amati et al. 2007) between isotropic energy, $E_{\mathrm{iso}}$, and peak energy, $E_{\text {peak }}$ (Ghisellini et al. 2006). GRB 060218 however is consistent with the the Amati relation, fuelling the debate that GRB 980425 and GRB 031203 may just be apparent outliers and their intrinsic properties may be consistent with the relations. Invoking a jet geometry, the low observed luminosities for these GRBs may be due to such factors as wider jet opening angle, variations in viewing angle such that the more off-axis a burst is viewed the lower its luminosity or profiled jets with the Lorentz factor decreasing off-axis (Yamazaki et al. 2003; Ramirez-Ruiz et al. 2005).

It has also been proposed that GRBs 980425 and 031203 are intrinsically sub-energetic events seen on-axis, based on afterglow observations (Soderberg et al. 2004) and within the constraints of the internal shock model (Daigne \& Mochkovitch 2007). Such inherently sub-luminous events would be permitted by the internal shock model, where the burst is produced by a mildly relativistic outflow. If intrinsically low-luminosity, the rate of bursts such as GRB 980425 may be far higher than that of "standard" cosmological GRBs but only detectable locally.

The possibility that low luminosity GRBs could be part of the same population as cosmological GRBs or form a separate sub-energetic population with a much higher rate has been considered (Soderberg et al. 2006; Cobb et al. 2006; Chapman et al. 2007; Guetta \& Della Valle 2007; Liang et al. 2007; Coward et al. 2008; Le \& Dermer 2007; Virgili et al. 2008). The large number of long-lag GRBs detected with IBIS favours the latter conclusion and indicates that low redshift GRBs are dominated by the low luminosity class.
The collapsar model can account for low-luminosity GRBs if they have low Lorentz factors and fail to produce highlyrelativistic jets due to baryon loading (Woosley \& MacFadyen 1999). It has also been proposed that the nature of the central engine may be different, e.g., a magnetar progenitor as opposed to a black hole (e.g. Soderberg et al. 2006; Toma et al. 2007). Some authors suggest that the $\gamma$-rays are produced in a supernova shock breakout (e.g. Matzner \& McKee 1999; Ghisellini et al. 2007). A very luminous X-ray outburst was observed from the core collapse SN in NGC 2770 (Soderberg et al. 2008) that may be the shock breakout as recently observed from a red supergiant in a galaxy in the COSMOS field (Schawinski et al. 2008) or an X-ray flash (Xu et al. 2008; Li 2008).

We evaluate the rate of GRBs over the whole sky using the 8 long-lag GRBs in the PCFoV (Table 4$)$ at $50 \%$ coding $(0.1 \mathrm{sr})$ over an exposure time of 4 years, adopting a distance of $250 \mathrm{Mpc}$ and assuming that 2 of the 8 GRBs are not at low redshift. We obtain $2640 \mathrm{Gpc}^{-3} \mathrm{yr}^{-1}$ which is $\sim 25 \%$ of the local rate of type Ib/c SNe (Soderberg et al. 2006). The major uncertainty in this estimate is the distance, where a change of only a factor of 2 increases or decreases this number by 8 to $21100 \mathrm{Gpc}^{-3} \mathrm{yr}^{-1}$ and $330 \mathrm{Gpc}^{-3} \mathrm{yr}^{-1}$, respectively.

The rate of low-luminosity GRBs at the adopted distance of $250 \mathrm{Mpc}$ exceeds the upper limit of $3 \%$ or $<300 \mathrm{Gpc}^{-3} \mathrm{yr}^{-1}$ of type Ib/c SN producing GRBs, which was derived assuming that all low luminosity GRBs would produce a SN and be as radio bright as the SN GRBs (Soderberg et al. 2006). However, the low luminosity GRB 060605 has no associated SN to faint limits and is evidence for a quiet end for some massive stars (Fynbo et al. 2006; Dado et al. 2006; McBreen et al. 2008). A GRB may occur without a corresponding SN being observed if the ${ }^{56} \mathrm{Ni}$ does not have sufficient energy to escape the black hole or if the progenitor star has a low angular momentum. The association of low luminosity GRBs with the supergalactic plane is not proof that they are associated with clusters of galaxies but indicates that clusters may play a role. It is interesting to note that the rate of type Ia $\mathrm{SNe}$ is higher in elliptical galaxies in clusters than in field ellipticals by a factor of $\sim 3$ (Mannucci et al. 2007). This effect is due to galaxy-galaxy interactions in clusters (Boselli \& Gavazzi 2006) either producing a small amount of young stars or affecting the evolution and properties of binary systems. In the latter case, there should also be an increase in the merger rate of white dwarfs or a white dwarf with a neutron star or black hole. A merger involving a white dwarf (Fryer et al. 1999; Middleditch 2004; Levan et al. 2006; King et al. 2007) should produce a long GRB that is likely to be fainter than the formation of a black hole in cosmological GRBs. There will be no supernova in the merger of a white dwarf with a neutron star or black hole, and probably a faint afterglow. In addition, the merger could take place in the intercluster region without a host galaxy if the binary is ripped from its host in the merger interaction involving the cluster galaxies (Niino \& Totani 2008).

\section{Conclusions}

INTEGRAL observations of gamma-ray bursts have yielded many interesting results and offer significant insight into the prompt $\gamma$-ray emission. IBAS successfully provides accurate, fast localisations of GRBs to the community at a rate of $\sim 0.8$ GRBs per month, enabling multi-wavelength afterglow observations to be carried out by other space-based missions and ground-based telescopes.

We have presented the spectral, spatial, and temporal lag analysis of the 47 INTEGRAL GRBs up to July 2007. Most 
weak INTEGRAL GRBs are well fit with a power law, while bright GRBs can be fit by both the Band and quasithermal models. Approximately $40 \%$ of the GRBs for which a lag was measured have long spectral lags ( $>0.75 \mathrm{~s}$ between $25-50$ and 50-300 keV). The long-lag GRBs are characterised by low peak flux, long slow pulses and are concentrated towards the supergalactic plane, reflecting the nearby large-scale structure of the Universe.

INTEGRAL therefore detects a large proportion of faint, long-lag GRBs that are inferred to be local. The sensitivity of IBIS is such that it can detect very faint GRBs, allowing INTEGRAL to probe the population of low-luminosity GRBs with long lags. This population appears to be distinct from that of high-luminosity GRBs and dominates the local GRB population.

Acknowledgements. The authors thank Darach Watson and Rob Preece for useful discussions and comments, Andreas von Kienlin for providing the GRB 060901 data and Erik Kuulkers for the INTEGRAL exposure map data. S.M.B. acknowledges the support of the European Union through a Marie Curie Intra-European Fellowship within the Sixth Framework Program.

\section{References}

Abraham, J., Abreu, P., Aglietta, M., et al. 2007, Science, 318, 938 Amati, L., Della Valle, M., Frontera, F., et al. 2007, A\&A, 463, 913 Band, D., Matteson, J., Ford, L., et al. 1993, ApJ, 413, 281

Band, D. L. 1997, ApJ, 486, 928

Beckmann, V., Borkowski, J., Courvoisier, T. J.-L., et al. 2003, A\&A, 411, L327 Boella, G., Butler, R. C., Perola, G. C., et al. 1997, A\&AS, 122, 299

Bolmont, J., Atteia, J. L., Jacholkowska, A., Piron, F., \& Pizzichini, G. 2006 [arXiv: astro-ph/0603725]

Boselli, A., \& Gavazzi, G. 2006, PASP, 118, 517

Bosnjak, Z., Celotti, A., Ghirlanda, G., Della Valle, M., \& Pian, E. 2006, A\&A, 447,121

Butler, N. R., Kocevski, D., \& Bloom, J. S. 2008 [arXiv: 0802 . 3396]

Campana, S., Mangano, V., Blustin, A. J., et al. 2006, Nature, 442, 1008

Castro-Tirado, A. J., Jelínek, M., Pandey, S. B., et al. 2006, A\&A, 459, 763

Castro-Tirado, A. J., Bremer, M., McBreen, S., et al. 2007, A\&A, 475, 101

Chapman, R., Tanvir, N. R., Priddey, R. S., \& Levan, A. J. 2007, MNRAS, 382, L21

Chen, L., Lou, Y.-Q., Wu, M., et al. 2005, ApJ, 619, 983

Cheng, L. X., Ma, Y. Q., Cheng, K. S., Lu, T., \& Zhou, Y. Y. 1995, A\&A, 300, 746

Cobb, B. E., Bailyn, C. D., van Dokkum, P. G., \& Natarajan, P. 2006, ApJ, 645, L113

Coward, D. M., Guetta, D., Burman, R. R., \& Imerito, A. 2008, MNRAS, 386, 111

Dado, S., Dar, A., De Rujula, A., \& Plaga, R. 2006 [arXiv:astro-ph/0611161]

Daigne, F., \& Mochkovitch, R. 2003, MNRAS, 342, 587

Daigne, F., \& Mochkovitch, R. 2007, A\&A, 465, 1

D’Avanzo, P., Fugazza, D., Melandri, A., et al. 2004, GCN, 2632

D’Avanzo, P., Malesani, D., Campana, S., et al. 2006 [arXiv: astro-ph/0612644]

de Luca, A., Melandri, A., Caraveo, P. A., et al. 2005, A\&A, 440, 85

Donaghy, T. Q., Lamb, D. Q., Sakamoto, T., et al. 2006 [arXiv:astro-ph/0605570]

Fenimore, E., Barthelmy, S., Cummings, J., et al. 2004, GCN, 2906

Filliatre, P., D'Avanzo, P., Covino, S., et al. 2005, A\&A, 438, 793

Filliatre, P., Covino, S., D’Avanzo, P., et al. 2006, A\&A, 448, 971

Fishman, G. J., \& Meegan, C. A. 1995, ARA\&A, 33, 415

Foley, R. J., Chen, H.-W., Bloom, J. S., \& Prochaska, J. X. 2005, GCN, 3483

Frail, D. A. 2005, GCN, 4350

Fryer, C. L., Woosley, S. E., Herant, M., \& Davies, M. B. 1999, ApJ, 520, 650

Fynbo, J. U., Jensen, B. L., Gorosabel, J., et al. 2001, A\&A, 369, 373

Fynbo, J. P. U., Watson, D., Thöne, C. C., et al. 2006, Nature, 444, 1047

Galama, T. J., Vreeswijk, P. M., van Paradijs, J., et al. 1998, Nature, 395, 670

Gehrels, N., Chincarini, G., Giommi, P., et al. 2004, ApJ, 611, 1005

Gehrels, N., Norris, J. P., Barthelmy, S. D., et al. 2006, Nature, 444, 1044

Ghirlanda, G., Celotti, A., \& Ghisellini, G. 2003, A\&A, 406, 879

Ghisellini, G., Ghirlanda, G., Mereghetti, S., et al. 2006, MNRAS, 372, 1699

Ghisellini, G., Ghirlanda, G., \& Tavecchio, F. 2007, MNRAS, 382, L77

Gonzalez-Riestra, R., \& Rodriguez-Pascual, P. 2004, GCN, 2533

Gorosabel, J., Lund, N., Brandt, S., Westergaard, N. J., \& Castro Cerón, J. M. 2004, A\&A, 427, 87
Götz, D., Mereghetti, S., Hurley, K., et al. 2003, A\&A, 409, 831 Gotz, D., Mereghetti, S., Paizis, A., et al. 2006, GCN, 5665 Grebenev, S. A., \& Chelovekov, I. V. 2007, Astron. Lett., 33, 789 Guetta, D., \& Della Valle, M. 2007, ApJ, 657, L73

Guetta, D., \& Piran, T. 2007, J. Cosmol. Astro-Part. Phys., 7, 3 Guetta, D., Perna, R., Stella, L., \& Vietri, M. 2004, ApJ, 615, L73 Guidorzi, C., Gomboc, A., Mundell, C. G., et al. 2006, GCN, 4504 Gupta, V., Das Gupta, P., \& Bhat, P. N. 2002 [arXiv: astro-ph/0206402] Hafizi, M., \& Mochkovitch, R. 2007, A\&A, 465, 67

Hakkila, J., \& Giblin, T. W. 2004, ApJ, 610, 361

Hakkila, J., Giblin, T. W., Young, K. C., et al. 2007, ApJS, 169, 62

Hakkila, J., Giblin, T. W., Norris, J. P., Fragile, P. C., \& Bonnell, J. T. 2008, ApJ, 677, L81

Hartmann, D. H., Briggs, M. S., \& Mannheim, K. 1996, in Am. Inst. Phys. Conf. Ser., 384, 397

Hurley, K., Mitrofanov, I., Kozyrev, A., et al. 2006, ApJS, 164, 124

Ioka, K., \& Nakamura, T. 2001, ApJ, 554, L163

Jakobsson, P., Hjorth, J., Fynbo, J. P. U., et al. 2004, ApJ, 617, L21

Kalemci, E., Boggs, S., Wunderer, C., \& Jean, P. 2004, in 5th INTEGRAL Workshop on the INTEGRAL Universe, 859

Kalemci, E., Boggs, S. E., Kouveliotou, C., Finger, M., \& Baring, M. G. 2007, ApJS, 169, 75

Kaneko, Y., Preece, R. D., Briggs, M. S., et al. 2006, ApJS, 166, 298

Kennea, J. A., Burrows, D. N., Zhang, B., \& Gehrels, N. 2005, GCN, 3426

Kennea, J. A., Godet, O., Burrows, D. N., \& Gehrels, N. 2006, GCN, 4624

King, A., Olsson, E., \& Davies, M. B. 2007, MNRAS, 374, L34

Kocevski, D., \& Liang, E. 2003, ApJ, 594, 385

Kommers, J. M., Lewin, W. H. G., Kouveliotou, C., et al. 2001, VizieR Online Data Catalog, 213, 40385

Kouveliotou, C., Meegan, C. A., Fishman, G. J., et al. 1993, ApJ, 413, L101

Labanti, C., Di Cocco, G., Ferro, G., et al. 2003, A\&A, 411, L149

Lahav, O., Santiago, B. X., Webster, A. M., et al. 2000, MNRAS, 312, 166

Lamb, D. Q. 2001, in Gamma-ray Bursts in the Afterglow Era, ed. E. Costa, F. Frontera, \& J. Hjorth, 297

Lapi, A., Kawakatu, N., Bosnjak, Z., et al. 2008 [arXiv:0802 . 0787]

Le, T., \& Dermer, C. D. 2007, ApJ, 661, 394

Lebrun, F., Leray, J. P., Lavocat, P., et al. 2003, A\&A, 411, L141

Lei, F., Dean, A. J., \& Hills, G. L. 1997, Space Sci. Rev., 82, 309

Levan, A. J., Wynn, G. A., Chapman, R., et al. 2006, MNRAS, 368, L1

Li, L.-X. 2008 [arXiv:astro-ph/0803.0079]

Liang, E., Zhang, B., Virgili, F., \& Dai, Z. G. 2007, ApJ, 662, 1111

Liang, E.-W., Zhang, B.-B., Stamatikos, M., et al. 2006, ApJ, 653, L81

Lloyd-Ronning, N. M., \& Petrosian, V. 2002, ApJ, 565, 182

Lund, N., Budtz-Jørgensen, C., Westergaard, N. J., et al. 2003, A\&A, 411, L231

Malaguti, G., Bazzano, A., Beckmann, V., et al. 2003, A\&A, 411, L307

Malesani, D., Tagliaferri, G., Chincarini, G., et al. 2004, ApJ, 609, L5

Mangano, V., Mineo, T., Parola, V. L., et al. 2005, GCN, 3564

Mannucci, F., Maoz, D., Sharon, K., et al. 2007, MNRAS, 382, 1121

Marcinkowski, R., Denis, M., Bulik, T., et al. 2006, A\&A, 452, 113

Mas-Hesse, J. M., Giménez, A., Culhane, J. L., et al. 2003, A\&A, 411, L261

Matzner, C. D., \& McKee, C. F. 1999, ApJ, 510, 379

McBreen, S., Quilligan, F., McBreen, B., Hanlon, L., \& Watson, D. 2001, A\&A, 380, L31

McBreen, S., Hanlon, L., McGlynn, S., et al. 2006, A\&A, 455, 433

McBreen, S., Foley, S., Watson, D., et al. 2008, ApJ, 677, L85

McGlynn, S., McBreen, S., Hanlon, L., et al. 2005, Nuovo Cimento C, 28, 481

McGlynn, S., Clark, D. J., Dean, A. J., et al. 2007, A\&A, 466, 895

McGlynn, S., Foley, S., McBreen, S., et al. 2008a, A\&A, submitted

McGlynn, S., et al. 2008b, in prep.

Mereghetti, S., Götz, D., Beckmann, V., et al. 2003a, A\&A, 411, L311

Mereghetti, S., Götz, D., Borkowski, J., Walter, R., \& Pedersen, H. 2003b, A\&A, 411, L291

Mereghetti, S., Götz, D., Tiengo, A., et al. 2003c, ApJ, 590, L73

Mereghetti, S., Götz, D., Andersen, M. I., et al. 2005, A\&A, 433, 113

Mereghetti, S., Paizis, A., Götz, D., \& Kreykenbohm, I. 2007, GCN, 6189

Mészáros, P. 2006, Rep. Progr. Phys., 69, 2259

Middleditch, J. 2004, ApJ, 601, L167

Mirabal, N., Halpern, J. P., An, D., Thorstensen, J. R., \& Terndrup, D. M. 2006, ApJ, 643, L99

Moran, L., Mereghetti, S., Götz, D., et al. 2005, A\&A, 432, 467

Niino, Y., \& Totani, T. 2008, ApJ, 677, L23

Norris, J., Barthelmy, S., Gehrels, N., et al. 2005, GCN, 3484

Norris, J. P. 2002, ApJ, 579, 386

Norris, J. P., \& Bonnell, J. T. 2006, ApJ, 643, 266

Norris, J. P., Marani, G. F., \& Bonnell, J. T. 2000, ApJ, 534, 248

Parola, V. L., Mangano, V., Cusumano, G., et al. 2005, GCN, 4347

Pellizza, L. J., Duc, P.-A., Le Floc'h, E., et al. 2006, A\&A, 459, L5

Piran, T. 2005, Rev. Mod. Phys., 76, 1143 
Piro, L., Frail, D. A., Gorosabel, J., et al. 2002, ApJ 577, 680

Preece, R. D., Briggs, M. S., Mallozzi, R. S., et al. 2000, ApJS, 126, 19

Prochaska, J. X., Bloom, J. S., Chen, H.-W., et al. 2004, ApJ, 611, 200

Prochaska, J. X., Ellison, S., Foley, R. J., \& Bloom, J. S. 2005, GCN, 3332

Quilligan, F., McBreen, B., Hanlon, L., et al. 2002, A\&A, 385, 377

Ramirez-Ruiz, E., Granot, J., Kouveliotou, C., et al. 2005, ApJ, 625, L91

Rau, A., Kienlin, A. V., Hurley, K., \& Lichti, G. G. 2005, A\&A, 438, 1175

Rees, M. J., \& Mészáros, P. 2005, ApJ, 628, 847

Rodriguez-Pascual, P., \& Gonzalez-Riestra, R. 2004, GCN, 2688

Rol, E., Wijers, R. A. M. J., Kouveliotou, C., Kaper, L., \& Kaneko, Y. 2005, ApJ, 624,868

Roming, P. W. A., Schady, P., Fox, D. B., et al. 2006, ApJ, 652, 1416

Ryde, F. 2005, ApJ, 625, L95

Ryde, F., Kocevski, D., Bagoly, Z., Ryde, N., \& Mészáros, A. 2005, A\&A, 432, 105

Ryde, F., Björnsson, C.-I., Kaneko, Y., et al. 2006, ApJ, 652, 1400

Sakamoto, T., Lamb, D. Q., Kawai, N., et al. 2005, ApJ, 629, 311

Salmonson, J. D. 2000, ApJ, 544, L115

Salmonson, J. D., \& Galama, T. J. 2002, ApJ, 569, 682

Salvaterra, R., Campana, S., Chincarini, G., Covino, S., \& Tagliaferri, G. 2008, MNRAS, 385, 189

Santos-Lleo, M., Calderon, P., \& Gotz, D. 2003, GCN, 2464

Sazonov, S. Y., Lutovinov, A. A., \& Sunyaev, R. A. 2004, Nature, 430, 646

Sazonov, S. Y., Lutovinov, A. A., Churazov, E. M., \& Sunyaev, R. A. 2006, Astron. Lett., 32, 297

Schaefer, B. E. 2004, ApJ, 602, 306

Schaefer, B. E. 2007, ApJ, 660, 16

Schawinski, K., Justham, S., Wolf, C., et al. 2008 [arXiv:0803.3596]

Shrader, C. R. 2006, in Gamma-Ray Bursts in the Swift Era, ed. S. S. Holt, N. Gehrels, \& J. A. Nousek, 836, 185
Soderberg, A. M., Kulkarni, S. R., Berger, E., et al. 2004, Nature, 430, 648 Soderberg, A. M., Kulkarni, S. R., Nakar, E., et al. 2006, Nature, 442, 1014 Soderberg, A. M., Berger, E., Page, K., et al. 2008 [arXiv : 0802 . 1712]

Stern, B. E., Tikhomirova, Y., Kompaneets, D., Svensson, R., \& Poutanen, J. 2001, ApJ, 563, 80

Stoughton, C., Lupton, R. H., Bernardi, M., et al. 2002, AJ, 123, 485

Taylor, G. B., Frail, D. A., Kulkarni, S. R., et al. 1998, ApJ, 502, L115

Tiengo, A., \& Mereghetti, S. 2006, A\&A, 449, 203

Tinney, C., Stathakis, R., Cannon, R., et al. 1998, IAU Circ., 6896

Toma, K., Ioka, K., Sakamoto, T., \& Nakamura, T. 2007, ApJ, 659, 1420

Ubertini, P., Lebrun, F., Di Cocco, G., et al. 2003, A\&A, 411, L131

Vaughan, S., Willingale, R., O’Brien, P. T., et al. 2004, ApJ, 603, L5

Vedrenne, G., Roques, J.-P., Schönfelder, V., et al. 2003, A\&A, 411, L63

Vergani, S. D., Romano, P., \& Guidorzi, C. 2007, GCN 6369

Vianello, G., Tiengo, A., \& Mereghetti, S. 2007, A\&A, 473, 423

Virgili, F., Liang, E., \& Zhang, B. 2008 [arXiv:0801.4751]

von Kienlin, A., Beckmann, V., Covino, S., et al. 2003a, A\&A, 411, L321

von Kienlin, A., Beckmann, V., Rau, A., et al. 2003b, A\&A, 411, L299

Watson, D., Hjorth, J., Levan, A., et al. 2004, ApJ, 605, L101

Watson, D., Vaughan, S. A., Willingale, R., et al. 2006, ApJ, 636, 967

Winkler, C., Courvoisier, T. J.-L., Di Cocco, G., et al. 2003, A\&A, 411, L1

Woosley, S. E., \& MacFadyen, A. I. 1999, A\&AS, 138, 499

Wu, B., \& Fenimore, E. 2000, ApJ, 535, L29

Xu, D., Zou, Y.-C., \& Fan, Y.-Z. 2008 [arXiv: astro-ph/0801.4325]

Yamazaki, R., Yonetoku, D., \& Nakamura, T. 2003, ApJ, 594, L79

Yi, T., Liang, E., Qin, Y., \& Lu, R. 2006, MNRAS, 367, 1751

Zhang, B., \& Mészáros, P. 2004, Int. J. Mod. Phys. A, 19, 2385

Zhang, Z., Xie, G. Z., Deng, J. G., \& Jin, W. 2006a, MNRAS, 373, 729

Zhang, Z.-B., Deng, J.-G., Lu, R.-J., \& Gao, H.-F. 2006b, Chin. J. Astron. Astrophys., 6, 312 


\section{Appendix A: INTEGRAL Lightcurves}

The lightcurves of 43 of the 47 INTEGRAL GRBs are presented in the 25-50 keV (dark lines) and 50-300 keV (light lines) energy bands. Exceptions are the XRFs which were not detected above $50 \mathrm{keV}$ and for which the lightcurves are plotted in the 15-25 keV (dark lines) and 25-50 keV (light lines) and the very weak GRBs and GRBs with telemetry gaps for which a lag could not be determined which are given over the full energy range of 25-300 keV. The regions which were used in the spectral lag analysis are denoted by solid vertical lines and the temporal resolution is that at which the lag was determined. All lightcurve data is from the IBIS instrument on board INTEGRAL with the exceptions of GRB 041219a and GRB 050525a for which data is taken from the BAT instrument on Swift due to IBIS telemetry saturation. A satellite slew occurred during GRB 030131 and the lightcurve is not presented here but is available in Götz et al. (2003). GRB 060428c was discovered in the INTEGRAL archival data and the lightcurve is available in Grebenev \& Chelovekov (2007). IBIS data is currently unavailable for GRB 060930 and GRB 070311. 
S. Foley et al.: Global characteristics of GRBs observed with INTEGRAL, Online Material p 2
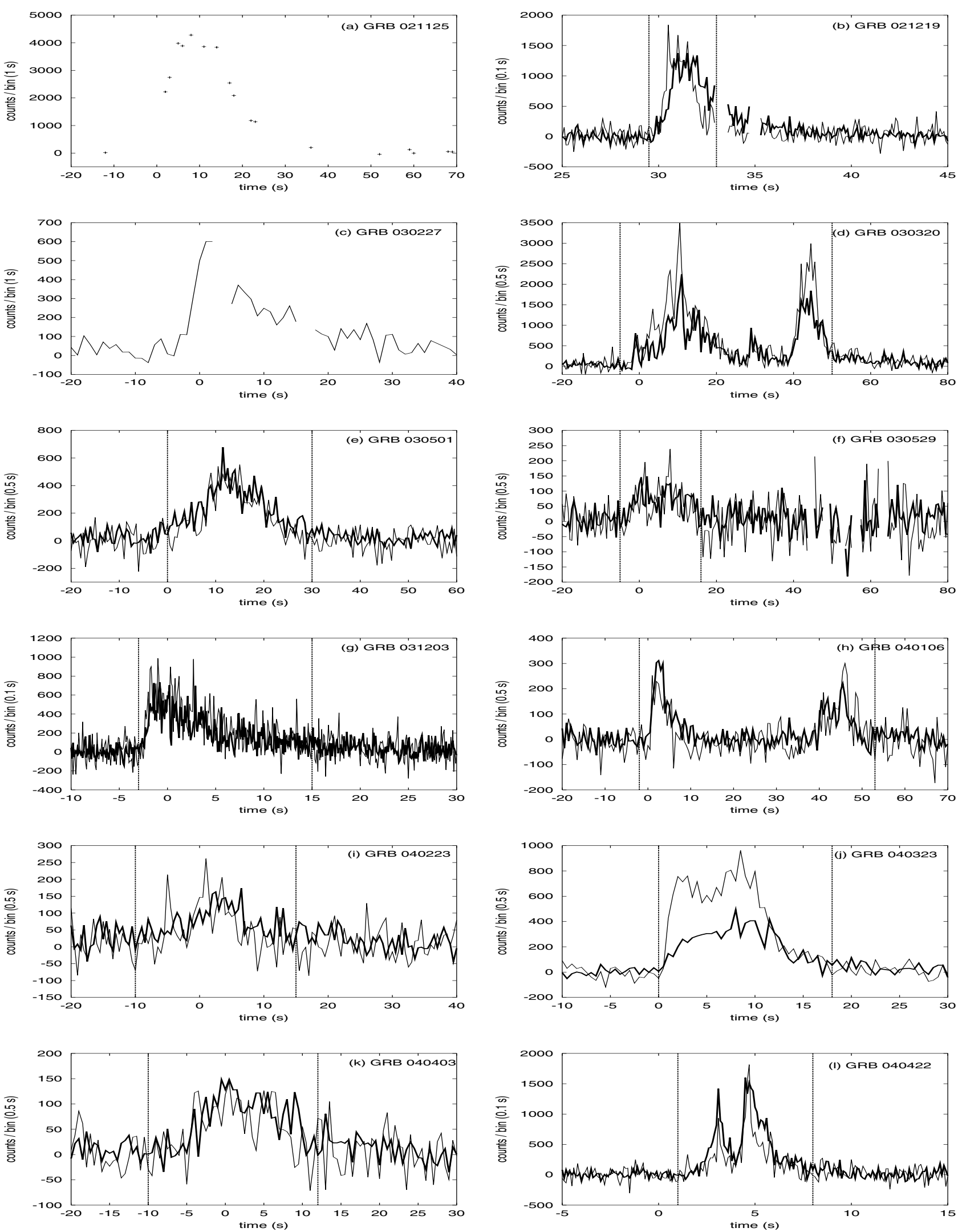

Fig. A.1. Lightcurves of GRBS observed with INTEGRAL. 
S. Foley et al.: Global characteristics of GRBs observed with INTEGRAL, Online Material p 3
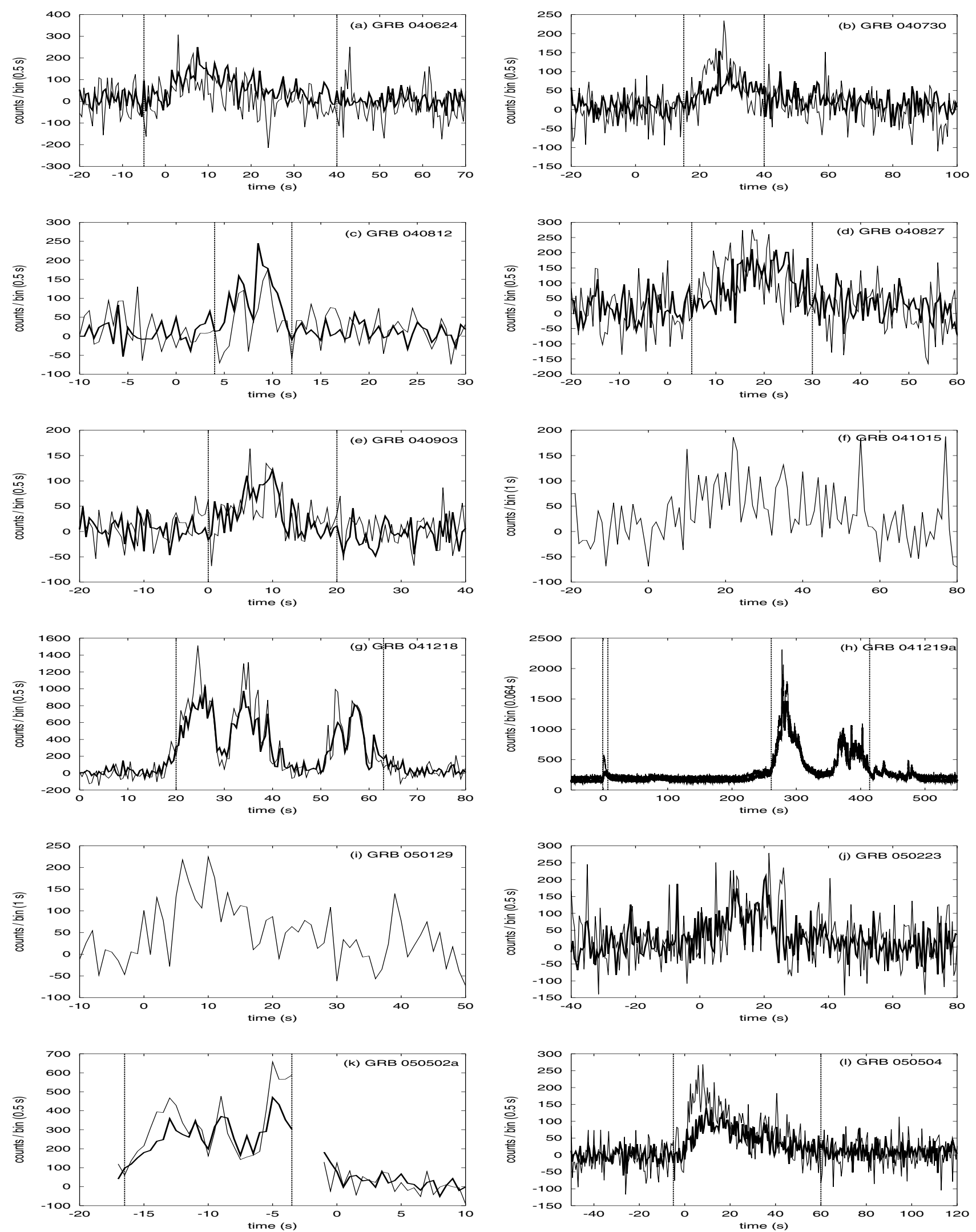

Fig. A.1. continued. 
S. Foley et al.: Global characteristics of GRBs observed with INTEGRAL, Online Material p 4
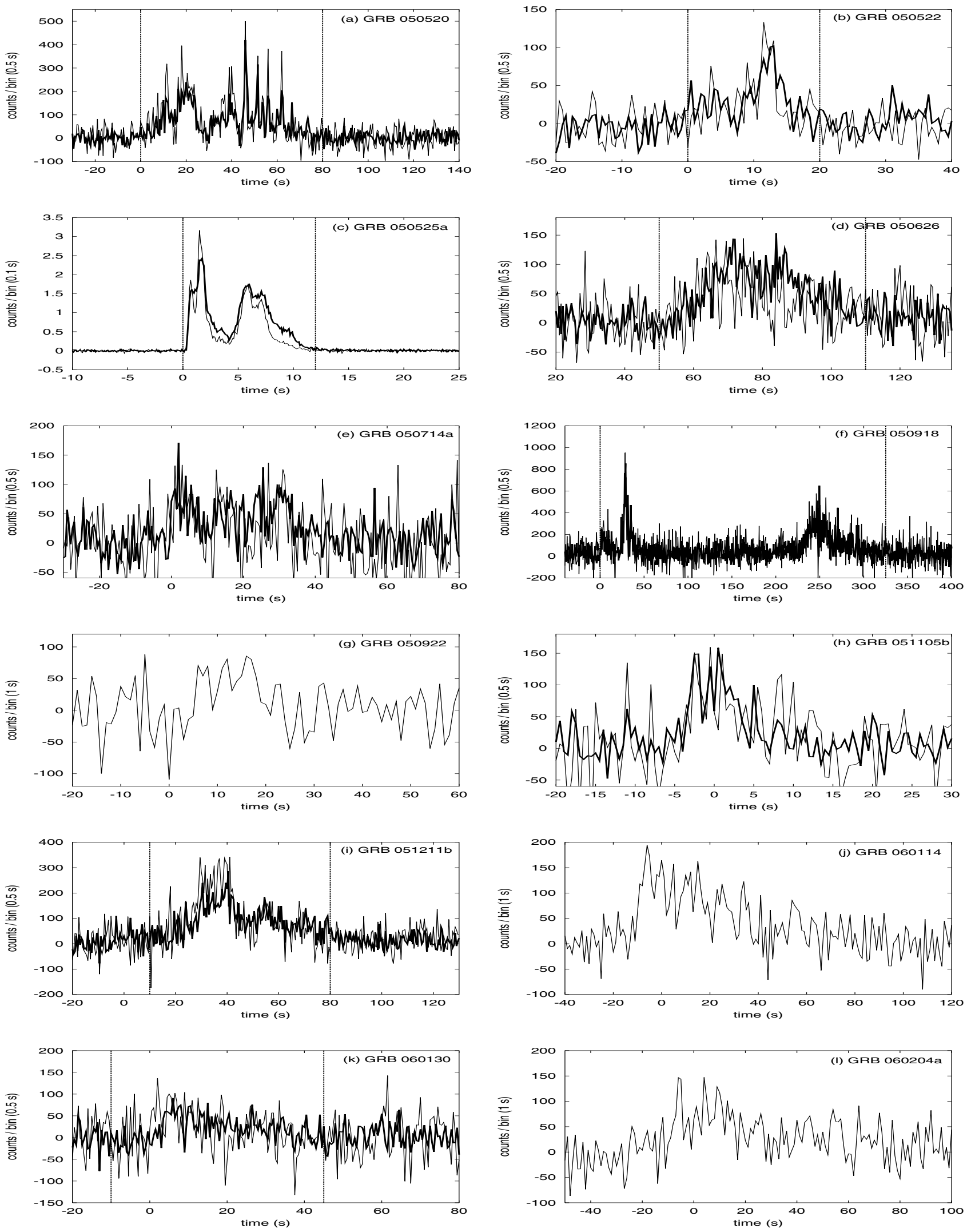

Fig. A.1. continued. 
S. Foley et al.: Global characteristics of GRBs observed with INTEGRAL, Online Material p 5
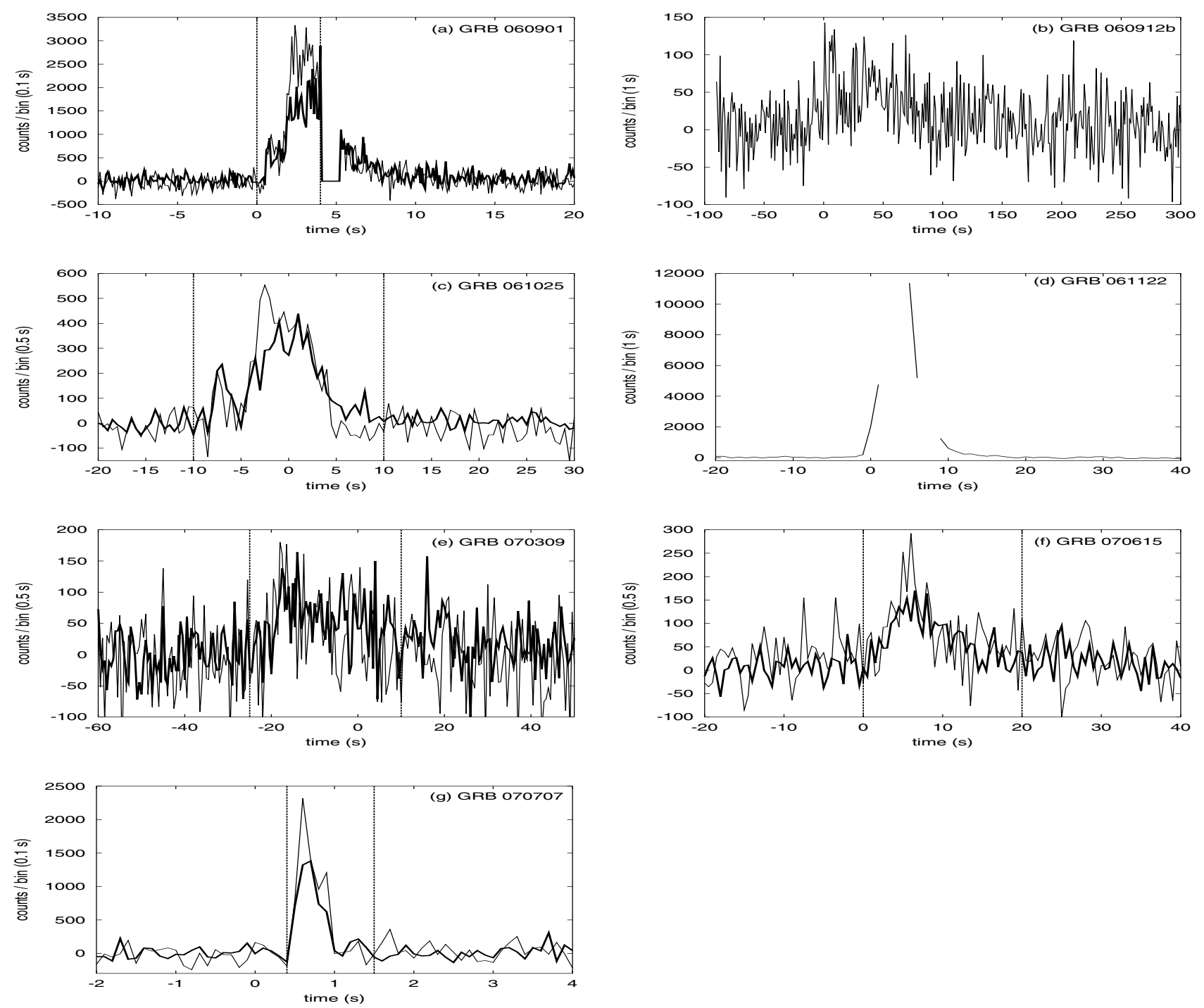

Fig. A.1. continued. 